\title{
Affine and Toric Hyperplane Arrangements
}

\author{
Richard Ehrenborg • Margaret Readdy • \\ MLE Slone
}

Received: 12 January 2007 / Revised: 28 April 2008 / Accepted: 24 December 2008 /

Published online: 4 March 2009

(C) Springer Science+Business Media, LLC 2009

\begin{abstract}
We extend the Billera-Ehrenborg-Readdy map between the intersection lattice and face lattice of a central hyperplane arrangement to affine and toric hyperplane arrangements. For arrangements on the torus, we also generalize Zaslavsky's fundamental results on the number of regions.
\end{abstract}

Keywords The cd-index - The complex of unbounded regions - Flag enumeration · Manifolds · The toric Zaslavsky invariant · The $n$-dimensional torus

\section{Introduction}

Traditionally combinatorialists study topological objects that are spherical, such as polytopes, or which are homeomorphic to a wedge of spheres, such as those obtained from shellable complexes. In this paper we break from this practice and study hyperplane arrangements on the $n$-dimensional torus.

It is classical that the convex hull of a finite collection of points in Euclidean space is a polytope and its boundary is a sphere. The key ingredient in this construction is convexity. At the moment there is no natural analogue of this process to obtain a complex whose geometric realization is a torus.

In this paper we are taking a zonotopal approach to working with arrangements on the torus. Recall that a zonotope can be defined without the notion of convexity,

R. Ehrenborg $(\bowtie) \cdot M$. Readdy $\cdot$ M. Slone

Department of Mathematics, University of Kentucky, Lexington, KY 40506-0027, USA

e-mail: jrge@ms.uky.edu

M. Readdy

e-mail: readdy@ms.uky.edu

M. Slone

e-mail: m.slone@uky.edu 
that is, it is a Minkowski sum of line segments. Dually, a central hyperplane arrangement gives rise to a spherical cell complex. By considering an arrangement on the torus, we are able to obtain a subdivision whose geometric realization is indeed the torus. We will see later in Sect. 3 that this amounts to restricting ourselves to arrangements whose subspaces in the Euclidean space $\mathbb{R}^{n}$ have coefficient matrices with rational entries. Under the quotient map $\mathbb{R}^{n} \rightarrow \mathbb{R}^{n} / \mathbb{Z}^{n}=T^{n}$, these subspaces are sent to subtori of the $n$-dimensional torus $T^{n}$.

Zaslavsky initiated the modern study of hyperplane arrangements in his fundamental treatise [47]. For early work in the field, see the references given in Grünbaum's text [28, Chap. 18]. Zaslavsky showed that evaluating the characteristic polynomial of a central hyperplane arrangement at -1 gives the number of regions in the complement of the arrangement. For central hyperplane arrangements, Bayer and Sturmfels [6] proved that the flag $f$-vector of the arrangement can be determined from the intersection lattice; see Theorem 2.3. However, their result is stated as a sum of chains in the intersection lattice, and hence it is hard to apply. Billera, Ehrenborg, and Readdy improved the Bayer-Sturmfels result by showing that it is enough to know the flag $f$-vector of the intersection lattice to compute the flag $f$-vector of a central arrangement. Recall that the cd-index of a regular cell complex is an efficient tool to encode its flag $f$-vector without linear redundancies [5]. The Billera-EhrenborgReaddy theorem gives an explicit way to compute the cd-index of the arrangement, and hence its flag $f$-vector [8].

We generalize Zaslavsky's theorem on the number of regions of a hyperplane arrangement to the toric case. Although there is no intersection lattice per se, one works with the intersection poset. From the Zaslavsky result we obtain a toric version of the Bayer-Sturmfels result for hyperplane arrangements, that is, there is a natural poset map from the face poset to the intersection poset, and furthermore, the cardinality of the inverse image of a chain under this map is described.

As in the case of a central hyperplane arrangement, our toric version of the BayerSturmfels result determines the flag $f$-vector of the face poset of a toric arrangement in terms of its intersection poset. However, this is far from being explicit. Using the coalgebraic techniques from [19], we are able to determine the flag $f$-vector explicitly in terms of the flag $f$-vector of the intersection poset. Moreover, the answer is given by a cd type of polynomial. The flag $f$-vector of a regular spherical complex is encoded by the cd-index, a noncommutative polynomial in the variables $\mathbf{c}$ and $\mathbf{d}$, whereas the $n$-dimensional toric analogue is a cd-polynomial plus the ab-polynomial $(\mathbf{a}-\mathbf{b})^{n+1}$.

Zaslavsky also showed that evaluating the characteristic polynomial of an affine arrangement at 1 gives the number of bounded regions in the complement of the arrangement. Thus we return to affine arrangements in Euclidean space with the twist that we study the unbounded regions. The unbounded regions form a spherical complex. In the case of central arrangements, this complex is exactly what was studied previously by Billera, Ehrenborg, and Readdy [8]. For noncentral arrangements, we determine the cd-index of this complex in terms of the lattice of unbounded intersections of the arrangement.

Interestingly, the techniques for studying toric arrangements and the unbounded complex of noncentral arrangements are similar. Hence, we present these results 
in the same paper. For example, the toric and noncentral analogues of the BayerSturmfels theorem only differ by which Zaslavsky invariant is used. The coalgebraic translations of the two analogues involve exactly the same argument, and the resulting underlying maps $\varphi_{t}$ (in the toric case) and $\varphi_{u b}$ (in the noncentral case) differ only slightly in their definitions.

We end with many open questions about subdivisions of manifolds.

\section{Preliminaries}

All the posets we will work with are graded, that is, have a unique minimal element $\hat{0}$, a unique maximal element $\hat{1}$, and rank function $\rho$. For two elements $x$ and $z$ in a graded poset $P$ such that $x \leq z$, let $[x, z]$ denote the interval $\{y \in P: x \leq y \leq z\}$. Observe that the interval $[x, z]$ is itself a graded poset. Given a graded poset $P$ of rank $n+1$ and $S \subseteq\{1, \ldots, n\}$, the $S$-rank-selected poset $P(S)$ is the poset consisting of the elements $P(S)=\{x \in P: \rho(x) \in S\} \cup\{\hat{0}, \hat{1}\}$. The partial order of $[x, y]$ and $P(S)$ are each inherited from that of $P$. For a graded poset $P$, let $P^{*}$ denote the dual poset, that is, the poset having the same underlying set as $P$ but with the order relation reversed: $x<_{P^{*}} y$ if and only if $y<_{P} x$. For standard poset terminology, we refer the reader to Stanley's work [41].

The Möbius function $\mu(x, y)$ on a poset $P$ is defined recursively by $\mu(x, x)=1$ and for elements $x, y \in P$ with $x<y$ by $\mu(x, y)=-\sum_{x \leq z<y} \mu(x, z)$; see Sect. 3.7 in [41]. For a graded poset $P$ with minimal element $\hat{0}$ and maximal element $\hat{1}$, we write $\mu(P)=\mu_{P}(\hat{0}, \hat{1})$.

We now review important results about hyperplane arrangements, the cd-index, and coalgebraic techniques. All are essential for proving the main results of this paper.

\subsection{Hyperplane Arrangements}

Let $\mathcal{H}=\left\{H_{1}, \ldots, H_{m}\right\}$ be a hyperplane arrangement in $\mathbb{R}^{n}$, that is, a finite collection of affine hyperplanes in $n$-dimensional Euclidean space $\mathbb{R}^{n}$. For brevity, throughout this paper we will often refer to a hyperplane arrangement as an arrangement. We call an arrangement essential if the normal vectors to the hyperplanes in $\mathcal{H}$ span $\mathbb{R}^{n}$. An arrangement that is not essential can be made essential by quotienting out by the subspace $V^{\perp}$ where $V$ is the subspace orthogonal to all of the hyperplanes in $\mathcal{H}$. In this paper we are only interested in essential arrangements.

Observe that the intersection $\bigcap_{i=1}^{m} H_{i}$ of all of the hyperplanes in an essential arrangement is either the empty set $\emptyset$ or a singleton point. We call an arrangement central if the intersection of all the hyperplanes is one point. We may assume that this point is the origin $\mathbf{0}$ and hence all of the hyperplanes are codimension 1 subspaces. If the intersection is the empty set, we call the arrangement noncentral.

The intersection lattice $\mathcal{L}$ is the lattice formed by ordering all the intersections of hyperplanes in $\mathcal{H}$ by reverse inclusion. If the intersection of all the hyperplanes in a given arrangement is empty, then we include the empty set $\emptyset$ as the maximal element in the intersection lattice. If the arrangement is central, the maximal element is $\{\mathbf{0}\}$. In all cases, the minimal element of $\mathcal{L}$ will be all of $\mathbb{R}^{n}$. 
For a hyperplane arrangement $\mathcal{H}$ with intersection lattice $\mathcal{L}$, the characteristic polynomial is defined by

$$
\chi(\mathcal{H} ; t)=\sum_{\substack{x \in \mathcal{L} \\ x \neq \emptyset}} \mu(\hat{0}, x) \cdot t^{\operatorname{dim}(x)},
$$

where $\mu$ denotes the Möbius function. The characteristic polynomial is a combinatorial invariant of the arrangement. The fundamental result of Zaslavsky [47] is that this invariant determines the number and type of regions.

Theorem 2.1 (Zaslavsky) For a hyperplane arrangement $\mathcal{H}$ in $\mathbb{R}^{n}$, the number of regions is given by $(-1)^{n} \cdot \chi(\mathcal{H} ; t=-1)$. Furthermore, the number of bounded regions is given by $(-1)^{n} \cdot \chi(\mathcal{H} ; t=1)$.

For a graded poset $P$, define the two Zaslavsky invariants $Z$ and $Z_{b}$ by

$$
\begin{aligned}
& Z(P)=\sum_{\hat{0} \leq x \leq \hat{1}}(-1)^{\rho(x)} \cdot \mu(\hat{0}, x), \\
& Z_{b}(P)=(-1)^{\rho(P)} \cdot \mu(P) .
\end{aligned}
$$

In order to work with Zaslavsky's result, we need the following reformulation of Theorem 2.1.

\section{Theorem 2.2}

(i) For a central hyperplane arrangement, the number of regions is given by $Z(\mathcal{L})$, where $\mathcal{L}$ is the intersection lattice of the arrangement.

(ii) For a noncentral hyperplane arrangement, the number of regions is given by $Z(\mathcal{L})-Z_{b}(\mathcal{L})$, where $\mathcal{L}$ is the intersection lattice of the arrangement. The number of bounded regions is given by $Z_{b}(\mathcal{L})$.

Given a central hyperplane arrangement $\mathcal{H}$, there are two associated lattices, namely, the intersection lattice $\mathcal{L}$ and the lattice $T$ of faces of the arrangement. The minimal element of $T$ is the empty set $\emptyset$, and the maximal element is the whole space $\mathbb{R}^{n}$. The lattice of faces can be seen as the face poset of the cell complex obtained by intersecting the arrangement $\mathcal{H}$ with a sphere of radius $R$ centered at the origin. Each hyperplane corresponds to a great circle on the sphere. An alternative way to view the lattice of faces $T$ is that the dual lattice $T^{*}$ is the face lattice of the zonotope corresponding to $\mathcal{H}$.

Let $\mathcal{L} \cup\{\hat{0}\}$ denote the intersection lattice with a new minimal element $\hat{0}$ adjoined. Define an order- and rank-preserving map $z$ from the dual lattice $T^{*}$ to the augmented lattice $\mathcal{L} \cup\{\hat{0}\}$ by sending a face of the arrangement, that is, a cone in $\mathbb{R}^{n}$, to its affine hull. Note that under the map $z$ the minimal element of $T^{*}$ is mapped to the minimal element of $\mathcal{L} \cup\{\hat{0}\}$. Observe that $z$ maps chains to chains. Hence we view $z$ as a map from the set of chains of $T^{*}$ to the set of chains of $\mathcal{L} \cup\{\hat{0}\}$. Bayer and Sturmfels [6] proved the following result about the inverse image of a chain under the map $z$. 
Theorem 2.3 (Bayer-Sturmfels) Let $\mathcal{H}$ be a central hyperplane arrangement with intersection lattice $\mathcal{L}$. Let $c=\left\{\hat{0}=x_{0}<x_{1}<\cdots<x_{k}=\hat{1}\right\}$ be a chain in $\mathcal{L} \cup\{\hat{0}\}$. Then the cardinality of the inverse image of the chain $c$ under the map $z: T^{*} \rightarrow$ $\mathcal{L} \cup\{\hat{0}\}$ is given by the product

$$
\left|z^{-1}(c)\right|=\prod_{i=2}^{k} Z\left(\left[x_{i-1}, x_{i}\right]\right)
$$

\subsection{The cd-index}

Let $P$ be a graded poset of rank $n+1$ with rank function $\rho$. For $S=\left\{s_{1}<\cdots<s_{k-1}\right\}$ a subset of $\{1, \ldots, n\}$, define $f_{S}$ to be the number of chains $c=\left\{\hat{0}=x_{0}<x_{1}<\cdots<\right.$ $\left.x_{k}=\hat{1}\right\}$ that have elements with ranks in the set $S$, that is,

$$
f_{S}=\left|\left\{c: \rho\left(x_{1}\right)=s_{1}, \ldots, \rho\left(x_{k-1}\right)=s_{k-1}\right\}\right| .
$$

Observe that $f_{S}$ is the number of maximal chains in the rank-selected poset $P(S)$. The flag $h$-vector is obtained by the relation (here we also present its inverse)

$$
h_{S}=\sum_{T \subseteq S}(-1)^{|S-T|} \cdot f_{T} \quad \text { and } \quad f_{S}=\sum_{T \subseteq S} h_{T} .
$$

Recall that by Philip Hall's theorem the Möbius function of the $S$-rank-selected poset $P(S)$ is given by $\mu(P(S))=(-1)^{|S|-1} \cdot h_{S}$.

Let $\mathbf{a}$ and $\mathbf{b}$ be two noncommutative variables each having degree 1 . For $S$ a subset of $\{1, \ldots, n\}$, let $u_{S}$ be the monomial $u_{S}=u_{1} \cdots u_{n}$, where $u_{i}=\mathbf{b}$ if $i \in S$ and $u_{i}=\mathbf{a}$ if $i \notin S$. Then the ab-index is the noncommutative polynomial defined by

$$
\Psi(P)=\sum_{S} h_{S} \cdot u_{S},
$$

where the sum is over all subsets $S \subseteq\{1, \ldots, n\}$. Observe that the ab-index of a poset $P$ of rank $n+1$ is a homogeneous polynomial of degree $n$.

A poset $P$ is Eulerian if every interval $[x, y]$, where $x<y$, satisfies the EulerPoincaré relation, that is, there are the same number of elements of odd as even rank. Equivalently, the Möbius function of $P$ is given by $\mu(x, y)=(-1)^{\rho(x, y)}$ for all $x \leq y$ in $P$. The quintessential result is that the ab-index of Eulerian posets has the following form.

Theorem 2.4 The ab-index of an Eulerian poset $P$ can be expressed in terms of the noncommutative variables $\mathbf{c}=\mathbf{a}+\mathbf{b}$ and $\mathbf{d}=\mathbf{a b}+\mathbf{b a}$.

This theorem was originally proved for face lattices of convex polytopes by Bayer and Klapper [5]. Stanley provided a proof for all Eulerian posets [43]. There are proofs which have both used and revealed the underlying algebraic structure. See for instance [15, 22]. When the ab-index $\Psi(P)$ is written in terms of $\mathbf{c}$ and $\mathbf{d}$, the resulting polynomial is called the cd-index. There are linear relations holding among 
the entries of the flag $f$-vector of an Eulerian poset, known as the generalized DehnSommerville relations; see [3]. The importance of the cd-index is that it removes all of these linear redundancies among the flag $f$-vector entries.

Observe that the variables $\mathbf{c}$ and $\mathbf{d}$ have degrees 1 and 2, respectively. Thus the cdindex of a poset of rank $n+1$ is a homogeneous polynomial of degree $n$ in the noncommutative variables $\mathbf{c}$ and $\mathbf{d}$. Define the reverse of an $\mathbf{a b}$-monomial $u=u_{1} u_{2} \cdots u_{n}$ to be $u^{*}=u_{n} \cdots u_{2} u_{1}$ and extend by linearity to an involution on $\mathbb{Z}\langle\mathbf{a}, \mathbf{b}\rangle$. Since $\mathbf{c}^{*}=\mathbf{c}$ and $\mathbf{d}^{*}=\mathbf{d}$, this involution applied to a cd-monomial simply reverses the cd-monomial. Finally, for a graded poset $P$, we have $\Psi(P)^{*}=\Psi\left(P^{*}\right)$.

A direct approach to describe the ab-index of a poset $P$ is to give each chain a weight and then sum over all chains. For a chain $c=\left\{\hat{0}=x_{0}<x_{1}<\cdots<x_{k}=\hat{1}\right\}$ in the poset $P$, define its weight to be

$$
\operatorname{wt}(c)=(\mathbf{a}-\mathbf{b})^{\rho\left(x_{0}, x_{1}\right)-1} \cdot \mathbf{b} \cdot(\mathbf{a}-\mathbf{b})^{\rho\left(x_{1}, x_{2}\right)-1} \cdot \mathbf{b} \cdots \mathbf{b} \cdot(\mathbf{a}-\mathbf{b})^{\rho\left(x_{k-1}, x_{k}\right)-1},
$$

where $\rho(x, y)$ denotes the rank difference $\rho(y)-\rho(x)$. The ab-index of $P$ is given by

$$
\Psi(P)=\sum_{c} \mathrm{wt}(c)
$$

where the sum is over all chains $c$ in the poset $P$.

Finally, a third description of the ab-index is Stanley's recursion for the ab-index of a graded poset [43, Eq. (7)]. It is:

$$
\Psi(P)=(\mathbf{a}-\mathbf{b})^{\rho(P)-1}+\sum_{\hat{0}<x<\hat{1}}(\mathbf{a}-\mathbf{b})^{\rho(x)-1} \cdot \mathbf{b} \cdot \Psi([x, \hat{1}]) .
$$

The initial condition for this recursion is the unique poset of rank $1, B_{1}$, where $\Psi\left(B_{1}\right)=1$.

\subsection{Coalgebraic Techniques}

A coproduct $\Delta$ on a free $\mathbb{Z}$-module $C$ is a linear map $\Delta: C \rightarrow C \otimes C$. In order to be explicit, we use the Sweedler notation [46] for writing the coproduct. To explain this notation, notice that $\Delta(w)$ is an element of $C \otimes C$ and thus has the form

$$
\Delta(w)=\sum_{i=1}^{k} w_{1}^{i} \otimes w_{2}^{i}
$$

where $k$ is the number of terms, and $w_{1}^{i}$ and $w_{2}^{i}$ belong to $C$. Since all the maps that are applied to $\Delta(w)$ treat each term the same, the Sweedler notation drops the index $i$, and one writes

$$
\Delta(w)=\sum_{w} w_{(1)} \otimes w_{(2)} .
$$

Informally, this sum should be thought of as all the ways of breaking the element $w$ in two pieces, where the first piece is denoted by $w_{(1)}$ and the second by $w_{(2)}$. 
The Sweedler notation for the expression $(\Delta \otimes \mathrm{id}) \circ \Delta$, where id denotes the identity map, is the following:

$$
((\Delta \otimes \mathrm{id}) \circ \Delta)(w)=\sum_{w} \sum_{w_{(1)}} w_{(1,1)} \otimes w_{(1,2)} \otimes w_{(2)} .
$$

The right-hand side should be thought of as first breaking $w$ into the two pieces $w_{(1)}$ and $w_{(2)}$ and then breaking $w_{(1)}$ into the two pieces $w_{(1,1)}$ and $w_{(1,2)}$. See Joni and Rota for a more detailed explanation [30].

The coproduct $\Delta$ is coassociative if $(\Delta \otimes$ id $) \circ \Delta=($ id $\otimes \Delta) \circ \Delta$. The Sweedler notation expresses coassociativity as

$$
\sum_{w} \sum_{w_{(1)}} w_{(1,1)} \otimes w_{(1,2)} \otimes w_{(2)}=\sum_{w} \sum_{w_{(2)}} w_{(1)} \otimes w_{(2,1)} \otimes w_{(2,2)} .
$$

Informally coassociativity states that all the possible ways to break $w$ into two pieces and then breaking the first piece into two pieces is equivalent to all the ways to break $w$ into two pieces and then break the second piece into two pieces. Compare coassociativity with associativity of a multiplication map $m: A \otimes A \rightarrow A$ on an algebra $A$.

Assuming coassociativity, Sweedler notation simplifies to

$$
\Delta^{2}(w)=\sum_{w} w_{(1)} \otimes w_{(2)} \otimes w_{(3)},
$$

where $\Delta^{2}$ is defined as $(\Delta \otimes \mathrm{id}) \circ \Delta=(\mathrm{id} \otimes \Delta) \circ \Delta$, and the three pieces have been renamed as $w_{(1)}, w_{(2)}$, and $w_{(3)}$. Coassociativity allows one to define the $k$-ary coproduct $\Delta^{k-1}: C \rightarrow C^{\otimes k}$ by the recursion $\Delta^{0}=$ id and $\Delta^{k}=\left(\Delta^{k-1} \otimes \mathrm{id}\right) \circ \Delta$. The Sweedler notation for the $k$-ary coproduct is

$$
\Delta^{k-1}(w)=\sum_{w} w_{(1)} \otimes w_{(2)} \otimes \cdots \otimes w_{(k)}
$$

Let $\mathbb{Z}\langle\mathbf{a}, \mathbf{b}\rangle$ denote the polynomial ring in the noncommutative variables $\mathbf{a}$ and $\mathbf{b}$. We define a coproduct $\Delta$ on the algebra $\mathbb{Z}\langle\mathbf{a}, \mathbf{b}\rangle$ by letting $\Delta$ satisfy the following identities: $\Delta(1)=0, \Delta(\mathbf{a})=\Delta(\mathbf{b})=1 \otimes 1$, and the Newtonian condition

$$
\Delta(u \cdot v)=\sum_{u} u_{(1)} \otimes u_{(2)} \cdot v+\sum_{v} u \cdot v_{(1)} \otimes v_{(2)} .
$$

For an ab-monomial $u=u_{1} u_{2} \cdots u_{n}$, we have that

$$
\Delta(u)=\sum_{i=1}^{n} u_{1} \cdots u_{i-1} \otimes u_{i+1} \cdots u_{n}
$$

The fundamental result for this coproduct is that the ab-index is a coalgebra homomorphism [19]. We express this result as the following identity. 
Theorem 2.5 (Ehrenborg-Readdy) For a graded poset $P$ with ab-index $w=\Psi(P)$ and for any $k$-multilinear map $M$ on $\mathbb{Z}\langle\mathbf{a}, \mathbf{b}\rangle$, the following coproduct identity holds:

$\sum_{c} M\left(\Psi\left(\left[x_{0}, x_{1}\right]\right), \Psi\left(\left[x_{1}, x_{2}\right]\right), \ldots, \Psi\left(\left[x_{k-1}, x_{k}\right]\right)\right)=\sum_{w} M\left(w_{(1)}, w_{(2)}, \ldots, w_{(k)}\right)$,

where the first sum is over all chains $c=\left\{\hat{0}=x_{0}<x_{1}<\cdots<x_{k}=\hat{1}\right\}$ of length $k$, and the second sum is the Sweedler notation of the $k$-ary coproduct of $w$, that is, $\Delta^{k-1}$.

\subsection{The cd-index of the Face Poset of a Central Arrangement}

We recall the definition of the omega map [8].

Definition 2.6 The linear map $\omega$ from $\mathbb{Z}\langle\mathbf{a}, \mathbf{b}\rangle$ to $\mathbb{Z}\langle\mathbf{c}, \mathbf{d}\rangle$ is formed by first replacing every occurrence of $\mathbf{a b}$ in a given $\mathbf{a b}$-monomial by $2 \mathbf{d}$ and then replacing the remaining letters by $\mathbf{c}$.

For a central hyperplane arrangement $\mathcal{H}$, the cd-index of the face poset is computed as follows [8].

Theorem 2.7 (Billera-Ehrenborg-Readdy) Let $\mathcal{H}$ be a central hyperplane arrangement with intersection lattice $\mathcal{L}$ and face lattice $T$. Then the cd-index of the face lattice $T$ is given by

$$
\Psi(T)=\omega(\mathbf{a} \cdot \Psi(\mathcal{L}))^{*}
$$

We review the basic ideas behind the proof of this theorem. We will refer back to them when we prove similar results for toric and affine arrangements in Sects. 3 and 4.

Define three linear operators $\kappa, \beta$, and $\eta$ on $\mathbb{Z}\langle\mathbf{a}, \mathbf{b}\rangle$ by

$$
\begin{aligned}
& \kappa(v)= \begin{cases}(\mathbf{a}-\mathbf{b})^{m} & \text { if } v=\mathbf{a}^{m} \text { for some } m \geq 0, \\
0 & \text { otherwise, }\end{cases} \\
& \beta(v)= \begin{cases}(\mathbf{a}-\mathbf{b})^{m} & \text { if } v=\mathbf{b}^{m} \text { for some } m \geq 0, \\
0 & \text { otherwise, }\end{cases}
\end{aligned}
$$

and

$$
\eta(v)= \begin{cases}2 \cdot(\mathbf{a}-\mathbf{b})^{m+k} & \text { if } v=\mathbf{b}^{m} \mathbf{a}^{k} \text { for some } m, k \geq 0, \\ 0 & \text { otherwise. }\end{cases}
$$

Observe that $\kappa$ and $\beta$ are both algebra maps. The following relations hold for a poset $P$ (see [8, Sect. 5]):

$$
\kappa(\Psi(P))=(\mathbf{a}-\mathbf{b})^{\rho(P)-1},
$$




$$
\begin{aligned}
& \beta(\Psi(P))=Z_{b}(P) \cdot(\mathbf{a}-\mathbf{b})^{\rho(P)-1}, \\
& \eta(\Psi(P))=Z(P) \cdot(\mathbf{a}-\mathbf{b})^{\rho(P)-1} .
\end{aligned}
$$

For $k \geq 1$, the operator $\varphi_{k}$ is defined by the coalgebra expression

$$
\varphi_{k}(v)=\sum_{v} \kappa\left(v_{(1)}\right) \cdot \mathbf{b} \cdot \eta\left(v_{(2)}\right) \cdot \mathbf{b} \cdots \mathbf{b} \cdot \eta\left(v_{(k)}\right),
$$

where the coproduct splits $v$ into $k$ parts. Finally, $\varphi$ is defined as the sum

$$
\varphi(v)=\sum_{k \geq 1} \varphi_{k}(v)
$$

Note that in this expression only a finite number of terms are nonzero. The connection with hyperplane arrangements is given by the following proposition.

Proposition 2.8 The $\mathbf{a b}$-index of the lattice of faces of a central hyperplane arrangement is given by

$$
\Psi(T)=\varphi(\Psi(\mathcal{L} \cup\{\hat{0}\}))^{*}
$$

The function $\varphi$ satisfies the functional equation

$$
\varphi(v)=\kappa(v)+\sum_{v} \varphi\left(v_{(1)}\right) \cdot \mathbf{b} \cdot \eta\left(v_{(2)}\right) .
$$

From this relation it follows that the function $\varphi$ satisfies the initial conditions $\varphi(1)=1$ and $\varphi(\mathbf{b})=2 \cdot \mathbf{b}$ and the recursions

$$
\begin{gathered}
\varphi(v \cdot \mathbf{a})=\varphi(v) \cdot \mathbf{c}, \\
\varphi(v \cdot \mathbf{b b})=\varphi(v \cdot \mathbf{b}) \cdot \mathbf{c}, \\
\varphi(v \cdot \mathbf{a b})=\varphi(v) \cdot 2 \mathbf{d},
\end{gathered}
$$

for an ab-monomial $v$; see [8, Sect. 5]. These recursions culminate in the following result.

Proposition 2.9 For an ab-monomial $w$ that begins with a, the two maps $\varphi$ and $\omega$ coincide, that is, $\varphi(w)=\omega(w)$.

Finally, Theorem 2.7 follows by Proposition 2.9 and from the fact that $\Psi(\mathcal{L} \cup$ $\{\hat{0}\})=\mathbf{a} \cdot \Psi(\mathcal{L})$.

\subsection{Regular Subdivisions of Manifolds}

The face poset $P(\Omega)$ of a cell complex $\Omega$ is the set of all cells in $\Omega$ together with a minimal element $\hat{0}$ and a maximal element $\hat{1}$. One partially orders two cells $\tau$ and $\sigma$ by requiring that $\tau<\sigma$ if the cell $\tau$ is contained in $\bar{\sigma}$, the closure of $\sigma$. In 
order to define a regular cell complex, consider the cell complex $\Omega$ embedded in Euclidean space $\mathbb{R}^{n}$. This condition is compatible with toric cell complexes since the $n$-dimensional torus can be embedded in $2 n$-dimensional Euclidean space. Let $B^{n}$ denote the ball $\left\{x \in \mathbb{R}^{n}: x_{1}^{2}+\cdots+x_{n}^{2} \leq 1\right\}$, and let $S^{n-1}$ denote the sphere $\left\{x \in \mathbb{R}^{n}: x_{1}^{2}+\cdots+x_{n}^{2}=1\right\}$. A cell complex $\Omega$ is regular if (i) $\Omega$ consists of a finite number of cells, (ii) for every cell $\sigma$ of $\Omega$, the pair $(\bar{\sigma}, \bar{\sigma}-\sigma)$ is homeomorphic to a pair $\left(B^{k}, S^{k-1}\right)$ for some integer $k$, and (iii) the boundary $\bar{\sigma}-\sigma$ is the disjoint union of smaller cells in $\Omega$. See Sect. 3.8 in [41] for more details. For a discussion of regular cell complexes not embedded in $\mathbb{R}^{n}$, see [10].

The face poset of a regular subdivision of the sphere is an Eulerian face poset and hence has a cd-index. For regular subdivisions of compact manifolds, a similar result holds. This was independently observed by Ed Swartz [45].

Theorem 2.10 Let $\Omega$ be a regular cell complex whose geometric realization is a compact $n$-dimensional manifold $M$. Let $\chi(M)$ denote the Euler characteristic of $M$. Then the ab-index of the face poset $P$ of $\Omega$ has the following form.

(i) If $n$ is odd, then $P$ is an Eulerian poset and hence $\Psi(P)$ can be written in terms of $\mathbf{c}$ and $\mathbf{d}$.

(ii) If $n$ is even, then $\Psi(P)$ has the form

$$
\Psi(P)=\left(1-\frac{\chi(M)}{2}\right) \cdot(\mathbf{a}-\mathbf{b})^{n+1}+\frac{\chi(M)}{2} \cdot \mathbf{c}^{n+1}+\Phi,
$$

where $\Phi$ is a homogeneous cd-polynomial of degree $n+1$ and $\Phi$ does not contain the term $\mathbf{c}^{n+1}$.

Proof Observe that the poset $P$ has rank $n+2$. By [41, Theorem 3.8.9] we know that every interval $[x, y]$ strictly contained in $P$ is Eulerian. When the rank of $P$ is odd, this implies that $P$ is also Eulerian; see [41, Exercise 69c]. Hence in this case the abindex of $P$ can be expressed as a cd-index. When $n$ is even, we use [15, Theorem 4.2] to conclude that the ab-index of $P$ belongs to $\mathbb{R}\left\langle\mathbf{c}, \mathbf{d},(\mathbf{a}-\mathbf{b})^{n+1}\right\rangle$. Since $\Psi(P)$ has degree $n+1$, the ab-index $\Psi(P)$ can be written in the form

$$
\Psi(P)=c_{1} \cdot(\mathbf{a}-\mathbf{b})^{n+1}+c_{2} \cdot \mathbf{c}^{n+1}+\Phi,
$$

where $\Phi$ is a homogeneous cd-polynomial of degree $n+1$ that does not contain any $\mathbf{c}^{n+1}$ terms. By looking at the coefficients of $\mathbf{a}^{n+1}$ and $\mathbf{b}^{n+1}$, we have $c_{1}+c_{2}=1$ and $c_{2}-c_{1}=\mu(P)=\chi(M)-1$, where the last identity is again [41, Theorem 3.8.9]. Solving for $c_{1}$ and $c_{2}$ proves the result.

Corollary 2.11 Let $P$ be the face poset of a regular cell complex whose geometric realization is a compact $n$-dimensional manifold $M$. If $n$ is odd, then the flag $h$-vector of $P$ is symmetric, that is, $h_{S}=h_{\bar{S}}$. If $n$ is even, the flag $h$-vector of $P$ satisfies

$$
h_{S}-h_{\bar{S}}=(-1)^{|S|} \cdot(2-\chi(M)) \text {. }
$$

For the $n$-dimensional torus, Theorem 2.10 can be expressed as follows. 
Corollary 2.12 Let $\Omega$ be a regular cell complex whose geometric realization is the $n$-dimensional torus $T^{n}$. Then the $\mathbf{a b}$-index of the face poset $P$ of $\Omega$ has the following form:

$$
\Psi(P)=(\mathbf{a}-\mathbf{b})^{n+1}+\Phi,
$$

where $\Phi$ is a homogeneous cd-polynomial of degree $n+1$ and $\Phi$ does not contain the term $\mathbf{c}^{n+1}$.

Proof When $n$ is even, this is Theorem 2.10. When $n$ is odd, this is Theorem 2.10 together with the two facts that $\chi\left(T^{n}\right)=0$ and $(\mathbf{a}-\mathbf{b})^{n+1}=\left(\mathbf{c}^{2}-2 \mathbf{d}\right)^{(n+1) / 2}$.

\section{Toric Arrangements}

\subsection{Toric Subspaces and Arrangements}

The $n$-dimensional torus $T^{n}$ is defined as the quotient $\mathbb{R}^{n} / \mathbb{Z}^{n}$. Recall that the torus $T^{n}$ is an Abelian group. When identifying the torus $T^{n}$ with the set $[0,1)^{n}$, the group structure is componentwise addition modulo 1 .

Lemma 3.1 Let $V$ be a $k$-dimensional affine subspace in $\mathbb{R}^{n}$ with rational coefficients. That is, $V$ has the form

$$
V=\left\{\vec{v} \in \mathbb{R}^{n}: A \vec{v}=\vec{b}\right\}
$$

where the matrix A has rational entries, and the vector $\vec{b}$ has real entries. Then the image of $V$ under the quotient map $\mathbb{R}^{n} \rightarrow \mathbb{R}^{n} / \mathbb{Z}^{n}$, denoted by $\bar{V}$, is a $k$-dimensional torus.

Proof By translating $V$, we may assume that the vector $\vec{b}$ is the zero vector, and therefore $V$ is a subspace. In this case, the intersection of $V$ with the integer lattice $\mathbb{Z}^{n}$ is a subgroup of the free Abelian group $\mathbb{Z}^{n}$. Since the matrix $A$ has all rational entries, the rank of this subgroup is $k$, that is, the subgroup is isomorphic to $\mathbb{Z}^{k}$. Hence the image $\bar{V}$ is the quotient $V /\left(V \cap \mathbb{Z}^{n}\right)$, which is isomorphic to the quotient $\mathbb{R}^{k} / \mathbb{Z}^{k}$, that is, a $k$-dimensional torus.

We call the image $\bar{V}$ a toric subspace of the torus $T^{n}$ because it is homeomorphic to some $k$-dimensional torus. When we remove the condition that the matrix $A$ is rational, the image is not necessarily homeomorphic to a torus.

The intersection of two toric subspaces is in general not a toric subspace but instead is the disjoint union of a finite number of toric subspaces. For two affine subspaces $V$ and $W$ with rational coefficients, we have that $\overline{V \cap W} \subseteq \bar{V} \cap \bar{W}$. In general, this containment is strict.

Define the translate of a toric subspace $U$ by a point $x$ on the torus to be the toric subspace $U+x=\{u+x: u \in U\}$. Alternatively, one may lift the toric subspace to 
an affine subspace in Euclidean space, translate it, and then map back to the torus. Then for two toric subspaces $V$ and $W$, their intersection has the form

$$
V \cap W=\bigcup_{p=1}^{r}\left(U+x_{p}\right),
$$

where $U$ is a toric subspace, $r$ is a nonnegative integer, and $x_{1}, \ldots, x_{r}$ are points on the torus $T^{n}$.

A toric hyperplane arrangement $\mathcal{H}=\left\{H_{1}, \ldots, H_{m}\right\}$ is a finite collection of toric hyperplanes. Define the intersection poset $\mathcal{P}$ of a toric arrangement to be the set of all connected components arising from all possible intersections of the toric hyperplanes, that is, all connected components of $\bigcap_{i \in S} H_{i}$ where $S \subseteq\{1, \ldots, m\}$, together with the empty set. Order the elements of the intersection poset $\mathcal{P}$ by reverse inclusion, that is, the torus $T^{n}$ is the minimal element of $\mathcal{P}$ corresponding to the empty intersection, and the empty set is the maximal element. A toric subspace $V$ is contained in the intersection poset $\mathcal{P}$ if there are toric hyperplanes $H_{i_{1}}, \ldots, H_{i_{k}}$ in the arrangement such that $V \subseteq H_{i_{1}} \cap \cdots \cap H_{i_{k}}$ and there is no toric subspace $W$ satisfying $V \subset W \subseteq H_{i_{1}} \cap \cdots \cap H_{i_{k}}$. In other words, $V$ has to be a maximal toric subspace in some intersection of toric hyperplanes from the arrangement.

The notion of using the intersection poset can be found in work of Zaslavsky, where he considers topological dissections [48]. In this setting there is not an intersection lattice, but rather an intersection poset.

To every toric hyperplane arrangement $\mathcal{H}=\left\{H_{1}, \ldots, H_{m}\right\}$, there is an associated periodic hyperplane arrangement $\widetilde{\mathcal{H}}$ in the Euclidean space $\mathbb{R}^{n}$. Namely, the inverse image of the toric hyperplane $H_{i}$ under the quotient map $\mathbb{R}^{n} \rightarrow \mathbb{R}^{n} / \mathbb{Z}^{n}$ is the union of parallel integer translates of a real hyperplane. Let $\widetilde{\mathcal{H}}$ be the collection of all these integer translates. Observe that every face of the toric arrangement $\mathcal{H}$ can be lifted to a parallel class of faces in the periodic real arrangement $\tilde{\mathcal{H}}$.

For a toric hyperplane arrangement $\mathcal{H}$, define the toric characteristic polynomial to be

$$
\chi(\mathcal{H} ; t)=\sum_{\substack{x \in \mathcal{P} \\ x \neq \emptyset}} \mu(\hat{0}, x) \cdot t^{\operatorname{dim}(x)} .
$$

Also for a toric hyperplane arrangement $\mathcal{H}$, define $T_{t}$ to be the face poset of the induced subdivision of the torus $T^{n}$. Note that $T_{t}$ is a graded poset of rank $n+2$ : the minimal element $\hat{0}$ is the empty face, the maximal element $\hat{1}$ is the torus, and the rank of the face $x$ is given by $\operatorname{dim}(x)+1$.

Example 3.2 Consider the line arrangement consisting of the two lines $y=2 \cdot x$ and $x=2 \cdot y$ in the plane $\mathbb{R}^{2}$. In $\mathbb{R}^{2}$ they intersect in one point, namely the origin, whereas on the torus $T^{2}$ they intersect in three points, namely $(0,0),(2 / 3,1 / 3)$, and $(1 / 3,2 / 3)$. The characteristic polynomial is given by $\chi(\mathcal{H} ; t)=t^{2}-2 \cdot t+3$. However, this arrangement is not regular, since the induced subdivision of $T^{2}$ is not regular. The boundary of each region is a wedge of two circles. See Fig. 1. 
Fig. 1 A toric line arrangement which subdivides the torus $T^{2}$ into a nonregular cell complex and its intersection poset

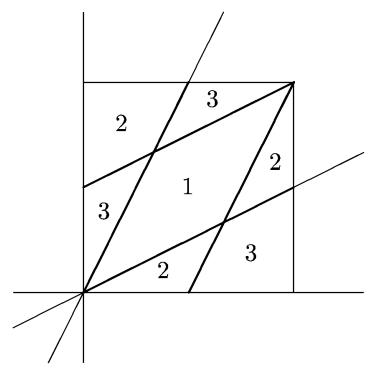

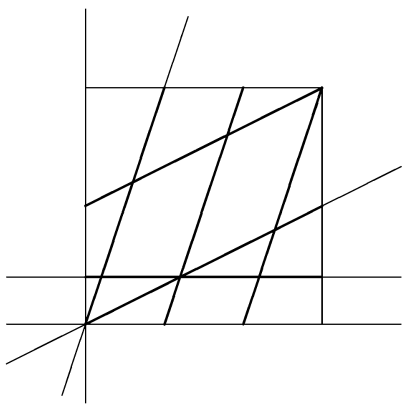

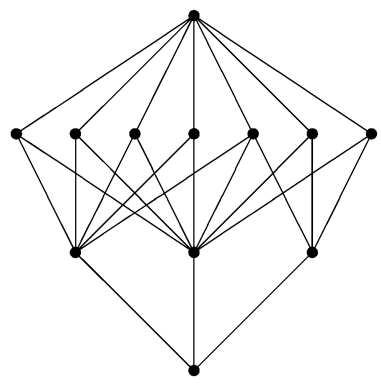

Fig. 2 A toric line arrangement and its intersection poset

Example 3.3 Consider the line arrangement consisting of the three lines $y=3 \cdot x$, $x=2 \cdot y$, and $y=1 / 5$. It subdivides the torus into a regular cell complex. The subdivision and the associated intersection poset are shown in Fig. 2. The characteristic polynomial is given by $\chi(\mathcal{H} ; t)=t^{2}-3 \cdot t+8$. Furthermore, the ab-index of the subdivision of the torus is given by $\Psi\left(T_{t}\right)=(\mathbf{a}-\mathbf{b})^{3}+7 \cdot \mathbf{d c}+8 \cdot \mathbf{c d}$, as the calculation in Table 1 shows.

Recall that $\mathbf{d c}=\mathbf{a b a}+\mathbf{a b b}+\mathbf{b a a}+\mathbf{b a b}$ and $\mathbf{c d}=\mathbf{a a b}+\mathbf{a b a}+\mathbf{b a b}+\mathbf{b b a}$. Here in the last three columns we indicate the contribution of a given term to each ab-monomial. Observe that the sum of the last three columns gives the flag $h$-vector entries.

We now give a natural interpretation of the toric characteristic polynomial. Recall that the intersection of toric subspaces is the disjoint union of toric subspaces that are translates of each other. Let $G$ be the collection of finite intersections of toric subspaces of the $n$-dimensional torus $T^{n}$, that is, $G$ consists of sets of the form $V=W_{1} \cap \cdots \cap W_{q}$, where $W_{1}, \ldots, W_{q}$ are toric subspaces. Such a set $V$ can be written as a union, more precisely, $V=\bigcup_{p=1}^{r}\left(U+x_{p}\right)$, where $U$ is a toric subspace, $r$ a nonnegative integer, and $x_{1}, \ldots, x_{r}$ are points on the torus. Observe that the empty set $\emptyset$ and the torus $T^{n}$ belong to $G$. Furthermore, $G$ is closed under finite intersections. Let $L$ be the distributive lattice consisting of all subsets of the torus $T^{n}$ that are obtained from the collection $G$ by finite intersections, finite unions, and complements. The set $G$ is the generating set for the lattice $L$. A valuation $v$ is a function on the lattice $L$ to an Abelian group satisfying $v(\emptyset)=0$ and $v(A)+v(B)=v(A \cap B)+v(A \cup B)$ for all sets $A, B \in L$. 
Table 1 Calculation of $\Psi\left(T_{t}\right)$ for the line arrangement in Fig. 2

\begin{tabular}{lrrlcll}
\hline$S$ & $f_{S}$ & $h_{S}$ & $u_{S}$ & $(\mathbf{a}-\mathbf{b})^{3}$ & $7 \cdot \mathbf{d c}$ & $8 \cdot \mathbf{c d}$ \\
\hline$\emptyset$ & 1 & 1 & aaa & 1 & 0 & 0 \\
$\{1\}$ & 7 & 6 & baa & -1 & 7 & 0 \\
$\{2\}$ & 15 & 14 & $\mathbf{a b a}$ & -1 & 7 & 8 \\
$\{3\}$ & 8 & 7 & $\mathbf{a a b}$ & -1 & 0 & 8 \\
$\{1,2\}$ & 30 & 9 & bba & 1 & 0 & 8 \\
$\{1,3\}$ & 30 & 16 & bab & 1 & 7 & 8 \\
$\{2,3\}$ & 30 & 8 & $\mathbf{a b b}$ & 1 & 7 & 0 \\
$\{1,2,3\}$ & 60 & -1 & bbb & -1 & 0 & 0 \\
\hline
\end{tabular}

The next theorem is analogous to Theorem 2.1 in [20]. The proof here is more involved due to the fact that the collection of toric subspaces is not closed under intersections.

Theorem 3.4 There is a valuation $v$ on the distributive lattice $L$ to $\mathbb{Z}[t]$ such that for a $k$-dimensional toric subspace $V$, its valuation is $v(V)=t^{k}$.

Proof Define the function $v$ on the generating set $G$ by

$$
v\left(\bigcup_{p=1}^{r}\left(U+x_{p}\right)\right)=r \cdot t^{k}
$$

where we assume that $U$ is a $k$-dimensional toric subspace, and the $r$ translates $U+$ $x_{1}, \ldots, U+x_{r}$ are pairwise disjoint. Observe that the function $v$ is additive with respect to disjoint unions, that is, for elements $V_{1}, \ldots, V_{m}$ in $G$ which are pairwise disjoint and $V_{1} \cup \cdots \cup V_{m} \in G$. In this case, each $V_{i}$ is a disjoint union of translates of the same affine subspace $U$, and both sides of the identity $v\left(V_{1}\right)+\cdots+v\left(V_{m}\right)=$ $v\left(V_{1} \cup \cdots \cup V_{m}\right)$ count the number of translates of $U$ times $t^{\operatorname{dim}(U)}$.

Groemer's integral theorem [27] (see also [35, Theorem 2.2.1]) states that a function $v$ defined on a generating set $G$ extends to a valuation on the distributive lattice generated by $G$ if for all $V_{1}, \ldots, V_{m}$ in $G$ such that $V_{1} \cup \ldots \cup V_{m} \in G$, the inclusionexclusion formula holds:

$$
v\left(V_{1} \cup \cdots \cup V_{m}\right)=\sum_{i} v\left(V_{i}\right)-\sum_{i<j} v\left(V_{i} \cap V_{j}\right)+\cdots .
$$

To verify this for our generating set $G$, first consider the case where the union $V_{1} \cup$ $\cdots \cup V_{m}$ is a toric subspace. This case implies that $V_{1} \cup \cdots \cup V_{m}=V_{i}$ for some index $i$. It then follows that the inclusion-exclusion formula (3.1) holds trivially.

Before considering the general case, we introduce some notation. For $S$ a nonempty subset of the index set $\{1, \ldots, m\}$, let $V_{S}=\bigcap_{i \in S} V_{i}$. Equation (3.1) can then be written as

$$
v\left(V_{1} \cup \cdots \cup V_{m}\right)=\sum_{S}(-1)^{|S|-1} \cdot v\left(V_{S}\right),
$$


where the sum ranges over nonempty subsets $S$ of $\{1, \ldots, m\}$. Now assume that $V_{1} \cup \cdots \cup V_{m}$ is the disjoint union $\left(U+x_{1}\right) \cup \cdots \cup\left(U+x_{r}\right)$. Let $V_{S, p}$ denote the intersection $V_{S} \cap\left(U+x_{p}\right)$. Observe that $U+x_{p}=\bigcup_{i=1}^{m} V_{\{i\}, p}$, and since $U+x_{p}$ is itself a toric subspace, we have already proved that the inclusion-exclusion formula (3.1) holds for this union. Hence we have

$$
\begin{aligned}
v\left(V_{1} \cup \cdots \cup V_{m}\right) & =\sum_{p=1}^{r} v\left(U+x_{p}\right) \\
& =\sum_{p=1}^{r} \sum_{S}(-1)^{|S|-1} \cdot v\left(V_{S, p}\right) \\
& =\sum_{S}(-1)^{|S|-1} \cdot \sum_{p=1}^{r} v\left(V_{S, p}\right) \\
& =\sum_{S}(-1)^{|S|-1} \cdot v\left(V_{S}\right),
\end{aligned}
$$

where $S$ ranges over all nonempty subsets of $\{1, \ldots, m\}$. The last step follows since the union $V_{S}=\bigcup_{p=1}^{r} V_{S, p}$ is pairwise disjoint.

By Möbius inversion we directly have the following theorem. The proof is standard. See the references [1, 11, 20, 31].

Theorem 3.5 The characteristic polynomial of a toric arrangement is given by

$$
\chi(\mathcal{H})=v\left(T^{n}-\bigcup_{i=1}^{m} H_{i}\right)
$$

When each region is an open ball, we can now determine the number of regions in a toric arrangement. The proof is analogous to the proofs in [20,21].

Theorem 3.6 Let $\mathcal{H}$ be a toric hyperplane arrangement on the $n$-dimensional torus $T^{n}$ that subdivides the torus into regions that are open $n$-dimensional balls. Then the number of regions in the complement of the arrangement is given by $(-1)^{n}$. $\chi(\mathcal{H} ; t=0)$.

Proof Observe that the Euler valuation $\varepsilon$ of a $k$-dimensional torus is given by the Kronecker delta $\delta_{k, 0}$. Hence the Euler valuation of a toric subspace $V$ of the $n$-dimensional torus $T^{n}$ is given by setting $t=0$ in the valuation $v(V)$, that is, $\varepsilon(V)=\left.v(V)\right|_{t=0}$. Since the two valuations $\varepsilon$ and $\left.v\right|_{t=0}$ are additive with respect to disjoint unions, they agree for any member of the generating set $G$. Hence they also agree for any member in the distributive lattice $L$. In particular, we have that

$$
\varepsilon\left(T^{n}-\bigcup_{i=1}^{m} H_{i}\right)=\left.v\left(T^{n}-\bigcup_{i=1}^{m} H_{i}\right)\right|_{t=0} .
$$


Since the Euler valuation of an open $n$-ball is $(-1)^{n}$ and $T^{n}-\bigcup_{i=1}^{m} H_{i}$ is a disjoint union of open balls, the left-hand side of (3.2) is $(-1)^{n}$ times the number of regions. The right-hand side is $\chi(\mathcal{H} ; t=0)$ by Theorem 3.5 .

Continuation of Example 3.2 Setting $t=0$ in the characteristic polynomial in Example 3.2, we obtain 3, which is indeed the number of regions of this arrangement.

We call a toric hyperplane arrangement $\mathcal{H}=\left\{H_{1}, \ldots, H_{m}\right\}$ rational if each hyperplane $H_{i}$ is of the form $\vec{a}_{i} \cdot \vec{x}=b_{i}$ where the vector $\vec{a}_{i}$ has integer entries, and $b_{i}$ is an integer for $1 \leq i \leq m$. This is equivalent to assuming that every constant $b_{i}$ is rational since every vector $\vec{a}_{i}$ was already assumed to be rational. In what follows it will be convenient to assume that every coefficient is integral in a given rational arrangement.

Define $\operatorname{lcm}(\mathcal{H})$ to be the least common multiple of all the $n \times n$ minors of the $n \times m$ matrix $\left(\vec{a}_{1}, \ldots, \vec{a}_{m}\right)$. We can now give a different interpretation of the toric characteristic polynomial by counting lattice points.

Theorem 3.7 For a rational hyperplane arrangement $\mathcal{H}$, there exists a constant $k$ such that for every $q>k$ where $q$ is a multiple of $\operatorname{lcm}(\mathcal{H})$, the toric characteristic polynomial evaluated at $q$ is given by the number of lattice points in $\left(\frac{1}{q} \mathbb{Z}\right)^{n} / \mathbb{Z}^{n}$ that do not lie on any of the toric hyperplanes $H_{i}$, that is,

$$
\chi(\mathcal{H} ; t=q)=\left|\left(\frac{1}{q} \mathbb{Z}\right)^{n} / \mathbb{Z}^{n}-\bigcup_{i=1}^{m} H_{i}\right| .
$$

The condition that $q$ is a multiple of $\operatorname{lcm}(\mathcal{H})$ implies that every subspace $x$ in the intersection poset $\mathcal{P}$ intersects the toric lattice $\left(\frac{1}{q} \mathbb{Z}\right)^{n} / \mathbb{Z}^{n}$ in exactly $q^{\operatorname{dim}(x)}$ points. Theorem 3.7 now follows by Möbius inversion. This theorem is the toric analogue of Athanasiadis' finite field method. See especially [2, Theorem 2.1].

In the case where $\operatorname{lcm}(\mathcal{H})=1$, the toric arrangement $\mathcal{H}$ is called unimodular. Novik, Postnikov, and Sturmfels [39] state Theorem 3.6 in the special case of unimodular arrangements. Their first proof is based upon Zaslavsky's result on the number of bounded regions in an affine arrangement. The second proof, due to Vic Reiner, is equivalent to our proof for arbitrary toric arrangements. See also the paper [48] by Zaslavsky, where more general arrangements are considered.

\subsection{Graphical Arrangements}

We digress in this subsection to discuss an application to graphical arrangements. For a graph $G$ on the vertex set $\{1, \ldots, n\}$, define the graphical arrangement $\mathcal{H}_{G}$ to be the collection of hyperplanes of the form $x_{i}=x_{j}$ for each edge $i j$ in the graph $G$.

Corollary 3.8 For a connected graph $G$ on $n$ vertices, the regions in the complement of the graphical arrangement $\mathcal{H}_{G}$ on the torus $T^{n}$ are each homotopy equivalent to the one-dimensional torus $T^{1}$. Furthermore, the number of regions is given by $(-1)^{n-1}$ times the linear coefficient of the chromatic polynomial of $G$. 
Proof Recall that the chromatic polynomial of the graph $G$ is equal to the characteristic polynomial of the graphical arrangement $\mathcal{H}_{G}$. The intersection lattice of the real arrangement $\mathcal{H}_{G}$ is the same as the intersection poset of the toric arrangement $\mathcal{H}_{G}$. Translating the graphical arrangement in the direction $(1, \ldots, 1)$ leaves the arrangement on the torus invariant. Since $G$ is connected, this is the only direction that leaves the arrangement invariant. Hence each region is homotopy equivalent to $T^{1}$. By adding the hyperplane $x_{1}=0$ to the arrangement we obtain a new arrangement $\mathcal{H}^{\prime}$ with the same number of regions, but with each region homeomorphic to a ball. Since the intersection lattice of $\mathcal{H}^{\prime}$ is just the Cartesian product of the two-element poset with the intersection lattice of $\mathcal{H}_{G}$, we have

$$
\chi\left(\mathcal{H}^{\prime}, t\right)=(t-1) \cdot \chi\left(\mathcal{H}_{G}, t\right) / t
$$

The number of regions is obtained by setting $t=0$ in this equality.

A similar statement holds for graphs that are disconnected. The result follows from the fact that the complement of the graphical arrangement is the product of the complements of each connected component.

Corollary 3.9 For a graph $G$ on $n$ vertices consisting of $k$ components, the regions in the complement of the graphical arrangement $\mathcal{H}_{G}$ on the torus $T^{n}$ are each homotopy equivalent to the $k$-dimensional torus $T^{k}$. The number of regions is given by $(-1)^{n-k}$ times the coefficient of $t^{k}$ in the chromatic polynomial of $G$.

Stanley [40] proved the celebrated result that the chromatic polynomial of a graph evaluated at $t=-1$ is $(-1)^{n}$ times the number of acyclic orientations of the graph. A similar interpretation for the linear coefficient of the chromatic polynomial is due to Greene and Zaslavsky [26]:

Theorem 3.10 (Greene-Zaslavsky) Let $G$ be a connected graph and $v$ a given vertex of the graph. The linear coefficient of the chromatic polynomial is $(-1)^{n-1}$ times the number of acyclic orientations of the graph such that the only sink is the vertex $v$.

Proof It is enough to give a bijection between regions in the complement of the graphical arrangement on the torus $T^{n}$ and acyclic orientations with the vertex $v$ as the unique sink. For a region $R$ of the arrangement, intersect it with the hyperplane $x_{v}=0$ to obtain the face $S$. Let $\mathcal{H}^{\prime}$ be the arrangement $\mathcal{H}_{G}$ together with the hyperplane $x_{v}=0$. Lift $S$ to a face $\widetilde{S}$ in the periodic arrangement $\widetilde{\mathcal{H}^{\prime}}$ in $\mathbb{R}^{n}$. Observe that $\widetilde{S}$ is the interior of a polytope. When minimizing the linear functional $L(x)=x_{1}+\cdots+x_{n}$ on the closure of the face $\widetilde{S}$, the optimum is a lattice point $k=\left(k_{1}, \ldots, k_{n}\right)$. Pick a point $x=\left(x_{1}, \ldots, x_{n}\right)$ in $\widetilde{S}$ close to the optimum, that is, each coordinate $x_{i}$ lies in the interval $\left[k_{i}, k_{i}+\epsilon\right)$ for some small $\epsilon>0$.

Let $y=\left(y_{1}, \ldots, y_{n}\right)$ be the image of the point $x$ on the torus $T^{n}$, that is, $y_{i} \equiv$ $x_{i} \bmod 1$. Note that each entry $y_{i}$ lies in the half open interval $[0,1)$ and that $y_{v}=0$. Construct an orientation of the graph $G$ by letting the edge $i j$ be oriented $i \rightarrow j$ if $y_{i}>y_{j}$. Note that this orientation is acyclic and has the vertex $v$ as a sink. 
To show that the vertex $v$ is the unique sink, assume that the vertex $i$ is also a sink, where $i \neq v$. In other words, for all neighbors $j$ of the vertex $i$, we have that $y_{i}<y_{j}$. We can continuously move the point $x$ in $\widetilde{S}$ by decreasing the value of the $i$ th coordinate $x_{i}$. Observe that there is no hyperplane in the periodic arrangement blocking the coordinate $x_{i}$ from passing through the integer value $k_{i}$ and continuing down to $k_{i}-1+\epsilon$. This contradicts the fact that we chose the original point $x$ close to the optimum of the linear functional $L$. Hence the vertex $i$ cannot be a sink.

It is straightforward to verify that this map from regions to the set of acyclic orientations with the unique sink at $v$ is a bijection.

The technique of assigning a point to every region of a toric arrangement using a linear functional was used by Novik, Postnikov, and Sturmfels in their paper [39]. See their first proof of the number of regions of a toric arrangement.

\subsection{The Toric Bayer-Sturmfels Result}

Define the toric Zaslavsky invariant of a graded poset $P$ having $\hat{0}$ and $\hat{1}$ by

$$
Z_{t}(P)=\sum_{x \text { coatom of } P}(-1)^{\rho(\hat{0}, x)} \cdot \mu(\hat{0}, x)=(-1)^{\rho(P)-1} \cdot \sum_{x \text { coatom of } P} \mu(\hat{0}, x) .
$$

We reformulate Theorem 3.6 as follows.

Theorem 3.11 For a toric hyperplane arrangement $\mathcal{H}$ on the torus $T^{n}$ that subdivides the torus into open $n$-dimensional balls, the number of regions is given by $Z_{t}(\mathcal{P})$, where $\mathcal{P}$ is the intersection poset of the arrangement $\mathcal{H}$.

As a corollary of Theorem 3.11, we can describe the $f$-vector of the subdivision $T_{t}$ of the torus. For similar results for more general manifolds, see [48, Sect. 3].

Corollary 3.12 The number of $i$-dimensional regions in the subdivision $T_{t}$ of the $n$-dimensional torus is given by the sum

$$
f_{i+1}\left(T_{t}\right)=(-1)^{i} \cdot \sum_{\substack{x \leq y \\ \operatorname{dim}(x)=i \\ \operatorname{dim}(y)=0}} \mu(x, y)
$$

where $\mu(x, y)$ denotes the Möbius function of the interval $[x, y]$ in the intersection poset $\mathcal{P}$.

Proof Each $i$-dimensional region is contained in a unique $i$-dimensional subspace $x$. By restricting the arrangement to the subspace $x$ and applying Theorem 3.6, we have that the number of $i$-dimensional regions in $x$ is given by $(-1)^{i} \cdot \sum_{x \leq y, \operatorname{dim}(y)=0} \mu(x, y)$. Summing over all $x$, the result follows.

For the remainder of this section, we will assume that the induced subdivision of the torus is a regular cell complex. Let $T_{t}$ be the face poset of the subdivision of the 
torus induced by the toric arrangement. Define the map $z_{t}: T_{t}^{*} \rightarrow \mathcal{P} \cup\{\hat{0}\}$ by sending each face to the smallest toric subspace in the intersection poset that contains the face and sending the minimal element in $T_{t}^{*}$ to $\hat{0}$. Observe that the map $z_{t}$ is order- and rank-preserving, as well as being surjective. As in the central hyperplane arrangement case, we view the map $z_{t}$ as a map from the set of chains of $T_{t}^{*}$ to the set of chains of $\mathcal{P} \cup\{\hat{0}\}$.

Let $x$ be an element in the intersection poset $\mathcal{P}$ of a toric hyperplane arrangement $\mathcal{H}$. Then the interval $[x, \hat{1}]$ is the intersection poset of a toric arrangement in the toric subspace $x$. The atoms of the interval $[x, \hat{1}]$ are the toric hyperplanes in this smaller toric arrangement.

More interesting is the geometric interpretation of the interval $[\hat{0}, x]$. It is the intersection lattice of a central hyperplane arrangement in $\mathbb{R}^{n-\operatorname{dim}(x)}$. Without loss of generality we may assume that $x$ contains the zero point $(0, \ldots, 0)$, that is, when we lift the toric subspace $x$ to an affine subspace $V$ in $\mathbb{R}^{n}$, we may assume that $V$ is a subspace of $\mathbb{R}^{n}$. Any toric subspace $y$ in the interval $[\hat{0}, x]$, that is, a toric subspace containing $x$, can be lifted to a subspace $W$ containing the subspace $V$. In particular, the toric hyperplanes in $[\hat{0}, x]$ lift to hyperplanes in $\mathbb{R}^{n}$ containing $V$. This lifting is a poset isomorphism, and we obtain an essential central arrangement of dimension $n-\operatorname{dim}(x)$ by quotienting out by the subspace $V$. We conclude by noticing that an interval $[x, y]$ in $\mathcal{P}$, where $y<\hat{1}$, is the intersection lattice of a central hyperplane arrangement.

The toric analogue of Theorem 2.3 is as follows.

Theorem 3.13 Let $\mathcal{P}$ be the intersection poset of a toric hyperplane arrangement whose induced subdivision is regular. Let $c=\left\{\hat{0}=x_{0}<x_{1}<\cdots<x_{k}=\hat{1}\right\}$ be a chain in $\mathcal{P} \cup\{\hat{0}\}$ with $k \geq 2$. Then the cardinality of the inverse image of the chain $c$ is given by the product

$$
\left|z_{t}^{-1}(c)\right|=\prod_{i=2}^{k-1} Z\left(\left[x_{i-1}, x_{i}\right]\right) \cdot Z_{t}\left(\left[x_{k-1}, x_{k}\right]\right) .
$$

Proof We need to count the number of ways we can select a chain $d=\left\{\hat{0}=y_{0}<\right.$ $\left.y_{1}<\cdots<y_{k}=\hat{1}\right\}$ in $T_{t}^{*}$ such that $z_{t}\left(y_{i}\right)=x_{i}$. The number of ways to select the element $y_{k-1}$ in $T_{t}^{*}$ is the number of regions in the arrangement restricted to the toric subspace $x_{k-1}$. By Theorem 3.11 this can be done in $Z_{t}\left(\left[x_{k-1}, x_{k}\right]\right)$ number of ways. Observe now that all other elements in the chain $d$ contain the face $y_{k-1}$.

To count the number of ways to select the element $y_{k-2}$, we follow the original argument of Bayer-Sturmfels. We would like to pick the face $y_{k-2}$ such that it contains the face $y_{k-1}$ and it is a region in the toric subspace $x_{k-2}$. This is equal to the number of regions in the central arrangement having the intersection lattice $\left[x_{k-2}, x_{k-1}\right]$, which is given by $Z\left(\left[x_{k-2}, x_{k-1}\right]\right)$. By iterating this procedure until we reach the element $y_{1}$, the result follows.

Corollary 3.14 The flag $f$-vector entry $f_{S}\left(T_{t}\right)$ of the face poset $T_{t}$ of a toric arrangement whose induced subdivision is a regular subdivision of $T^{n}$ is divisible by $2^{|S|-1}$ for $S \subseteq\{1, \ldots, n+1\}$ with $S \neq \emptyset$. 
Proof The proof follows from the fact that the Zaslavsky invariant $Z$ is an even integer and that a given flag $f$-vector entry is the appropriate sum of products appearing in Theorem 3.13.

\subsection{The Connection between Posets and Coalgebras}

For an ab-monomial $v$, define the linear map $\lambda_{t}$ by letting

$$
\lambda_{t}(v)= \begin{cases}(\mathbf{a}-\mathbf{b})^{m} & \text { if } v=\mathbf{b}^{m} \text { for some } m \geq 0, \\ (\mathbf{a}-\mathbf{b})^{m+1} & \text { if } v=\mathbf{b}^{m} \mathbf{a} \text { for some } m \geq 0, \\ 0 & \text { otherwise. }\end{cases}
$$

Define the linear operator $H^{\prime}$ on $\mathbb{Z}\langle\mathbf{a}, \mathbf{b}\rangle$ to be the one which removes the last letter in each ab-monomial, that is, $H^{\prime}(w \cdot \mathbf{a})=H^{\prime}(w \cdot \mathbf{b})=w$ and $H^{\prime}(1)=0$. We use the prime in the notation to distinguish it from the $H$ map defined in $[8$, Sect. 8], which instead removes the first letter in each ab-monomial. From [8] we have the following lemma.

Lemma 3.15 For a graded poset $P$ with $\hat{1}$ of rank greater than or equal to 2 , the following identity holds:

$$
H^{\prime}(\Psi(P))=\sum_{x \text { coatom of } P} \Psi([\hat{0}, x]) .
$$

The next lemma gives the relation between the toric Zaslavsky invariant $Z_{t}$ and the map $\lambda_{t}$.

Lemma 3.16 For a graded poset $P$ with $\hat{1}$ of rank greater than or equal to 1 , the following identity holds:

$$
\lambda_{t}(\Psi(P))=Z_{t}(P) \cdot(\mathbf{a}-\mathbf{b})^{\rho(P)-1} .
$$

Proof When $P$ has rank 1 , both sides are equal to 1 . For an ab-monomial $v$ different from 1 , we have that $\lambda_{t}(v)=\beta\left(H^{\prime}(v)\right) \cdot(\mathbf{a}-\mathbf{b})$. Hence

$$
\begin{aligned}
\lambda_{t}(\Psi(P)) & =\beta\left(H^{\prime}(\Psi(P))\right) \cdot(\mathbf{a}-\mathbf{b}) \\
& =\sum_{x \text { coatom of } P} \beta(\Psi([\hat{0}, x])) \cdot(\mathbf{a}-\mathbf{b}) \\
& =(-1)^{\rho(P)} \cdot \sum_{x \text { coatom of } P} \mu(\hat{0}, x) \cdot(\mathbf{a}-\mathbf{b})^{\rho(P)-1},
\end{aligned}
$$

which concludes the proof.

Define a sequence of functions $\varphi_{t, k}: \mathbb{Z}\langle\mathbf{a}, \mathbf{b}\rangle \rightarrow \mathbb{Z}\langle\mathbf{a}, \mathbf{b}\rangle$ by $\varphi_{t, 1}=\kappa$ and, for $k \geq 2$,

$$
\varphi_{t, k}(v)=\sum_{v} \kappa\left(v_{(1)}\right) \cdot \mathbf{b} \cdot \eta\left(v_{(2)}\right) \cdot \mathbf{b} \cdot \eta\left(v_{(3)}\right) \cdot \mathbf{b} \cdots \mathbf{b} \cdot \eta\left(v_{(k-1)}\right) \cdot \mathbf{b} \cdot \lambda_{t}\left(v_{(k)}\right) .
$$


Finally, let $\varphi_{t}(v)$ be the sum $\varphi_{t}(v)=\sum_{k \geq 1} \varphi_{t, k}(v)$.

Theorem 3.17 The ab-index of the face poset $T_{t}$ of a toric arrangement is given by

$$
\Psi\left(T_{t}\right)^{*}=\varphi_{t}(\Psi(\mathcal{P} \cup\{\hat{0}\})) .
$$

Proof The ab-index of the poset $T_{t}$ is given by the sum $\Psi\left(T_{t}\right)=\sum_{c}\left|z_{t}^{-1}(c)\right| \cdot \operatorname{wt}(c)$. Fix $k \geq 2$ and sum over all chains $c=\left\{\hat{0}=x_{0}<x_{1}<\cdots<x_{k}=\hat{1}\right\}$ of length $k$. We then have

$$
\begin{aligned}
& \sum_{c}\left|z_{t}^{-1}(c)\right| \cdot \operatorname{wt}(c) \\
&=\sum_{c} \prod_{i=2}^{k-1} Z\left(\left[x_{i-1}, x_{i}\right]\right) \cdot Z_{t}\left(\left[x_{k-1}, x_{k}\right]\right) \cdot(\mathbf{a}-\mathbf{b})^{\rho\left(x_{0}, x_{1}\right)-1} \\
& \cdot \mathbf{b} \cdots \mathbf{b} \cdot(\mathbf{a}-\mathbf{b})^{\rho\left(x_{k-1}, x_{k}\right)-1} \\
&= \sum_{c} \kappa\left(\Psi\left(\left[x_{0}, x_{1}\right]\right)\right) \cdot \prod_{i=2}^{k-1}\left(\mathbf{b} \cdot \eta\left(\Psi\left(\left[x_{i-1}, x_{i}\right]\right)\right)\right) \cdot \mathbf{b} \cdot \lambda_{t}\left(\Psi\left(\left[x_{k-1}, x_{k}\right]\right)\right) \\
&= \sum_{w} \kappa\left(w_{(1)}\right) \cdot \prod_{i=2}^{k-1}\left(\mathbf{b} \cdot \eta\left(w_{(i)}\right)\right) \cdot \mathbf{b} \cdot \lambda_{t}\left(w_{(k)}\right) \\
&= \varphi_{t, k}(w),
\end{aligned}
$$

where we let $w$ denote the ab-index of the augmented intersection poset $\mathcal{P} \cup\{\hat{0}\}$. For $k=1$, we have that $(\mathbf{a}-\mathbf{b})^{\rho\left(T_{t}\right)-1}=\varphi_{t, 1}(\Psi(\mathcal{P} \cup\{\hat{0}\}))$. Summing over all $k \geq 1$, we obtain the result.

3.5 Evaluating the Function $\varphi_{t}$

Proposition 3.18 For an ab-monomial $v$, the following identity holds:

$$
\varphi_{t}(v)=\kappa(v)+\sum_{v} \varphi\left(v_{(1)}\right) \cdot \mathbf{b} \cdot \lambda_{t}\left(v_{(2)}\right)
$$

Proof Using the coassociative identity $\Delta^{k-1}=\left(\Delta^{k-2} \otimes\right.$ id $) \circ \Delta$, for $k \geq 2$, we have that

$$
\begin{aligned}
\varphi_{t, k}(v) & =\sum_{v} \kappa\left(v_{(1)}\right) \cdot \mathbf{b} \cdot \eta\left(v_{(2)}\right) \cdot \mathbf{b} \cdots \mathbf{b} \cdot \eta\left(v_{(k-1)}\right) \cdot \mathbf{b} \cdot \lambda_{t}\left(v_{(k)}\right) \\
& =\sum_{v} \sum_{v_{(1)}} \kappa\left(v_{(1,1)}\right) \cdot \mathbf{b} \cdot \eta\left(v_{(1,2)}\right) \cdot \mathbf{b} \cdots \mathbf{b} \cdot \eta\left(v_{(1, k-1)}\right) \cdot \mathbf{b} \cdot \lambda_{t}\left(v_{(2)}\right) \\
& =\sum_{v} \varphi_{k-1}\left(v_{(1)}\right) \cdot \mathbf{b} \cdot \lambda_{t}\left(v_{(2)}\right) .
\end{aligned}
$$


By summing over all $k \geq 1$, the result follows.

Lemma 3.19 Let $v$ be an $\mathbf{a b - m o n o m i a l ~ t h a t ~ b e g i n s ~ w i t h ~} \mathbf{a}$, and let $x$ be either $\mathbf{a}$ or $\mathbf{b}$. Then

$$
\varphi_{t}(v \cdot \mathbf{a} \cdot x)=\kappa(v \cdot \mathbf{a} \cdot x)+1 / 2 \cdot \omega(v \cdot \mathbf{a b}) .
$$

Proof Using Proposition 3.18, we have

$$
\begin{aligned}
\varphi_{t}(v \cdot \mathbf{a} \cdot x)= & \kappa(v \cdot \mathbf{a} \cdot x)+\varphi(v \cdot \mathbf{a}) \cdot \mathbf{b} \cdot \lambda_{t}(1)+\varphi(v) \cdot \mathbf{b} \cdot \lambda_{t}(x) \\
& +\sum_{v} \varphi\left(v_{(1)}\right) \cdot \mathbf{b} \cdot \lambda_{t}\left(v_{(2)} \cdot \mathbf{b} \cdot x\right) \\
= & \kappa(v \cdot \mathbf{a} \cdot x)+\varphi(v) \cdot \mathbf{c} \cdot \mathbf{b}+\varphi(v) \cdot \mathbf{b} \cdot(\mathbf{a}-\mathbf{b}) \\
= & \kappa(v \cdot \mathbf{a} \cdot x)+\omega(v) \cdot \mathbf{d} \\
= & \kappa(v \cdot \mathbf{a} \cdot x)+1 / 2 \cdot \omega(v \cdot \mathbf{a b}),
\end{aligned}
$$

since $\lambda_{t}\left(v_{(2)} \cdot \mathbf{b} \cdot x\right)=0$.

Lemma 3.20 Let $v$ be an $\mathbf{a b - m o n o m i a l ~ t h a t ~ b e g i n s ~ w i t h ~} \mathbf{a}$, let $k$ be a positive integer, and let $x$ be either $\mathbf{a}$ or $\mathbf{b}$. Then the following evaluation holds:

$$
\varphi_{t}\left(v \cdot \mathbf{a b}^{k} \cdot x\right)=\kappa\left(v \cdot \mathbf{a b}^{k} \cdot x\right)+1 / 2 \cdot \omega\left(v \cdot \mathbf{a b}^{k+1}\right) .
$$

Proof Using Proposition 3.18, we have

$$
\begin{aligned}
& \varphi_{t}\left(v \cdot \mathbf{a b}^{k} \cdot x\right)-\kappa\left(v \cdot \mathbf{a b}^{k} \cdot x\right) \\
& =\varphi\left(v \cdot \mathbf{a b}^{k}\right) \cdot \mathbf{b} \cdot \lambda_{t}(1)+\varphi(v \cdot \mathbf{a}) \cdot \mathbf{b} \cdot \lambda_{t}\left(\mathbf{b}^{k-1} \cdot x\right) \\
& +\varphi(v) \cdot \mathbf{b} \cdot \lambda_{t}\left(\mathbf{b}^{k} \cdot x\right)+\sum_{i+j=k-2} \varphi\left(v \cdot \mathbf{a b} \mathbf{b}^{i+1}\right) \cdot \mathbf{b} \cdot \lambda_{t}\left(\mathbf{b}^{j} \cdot x\right) \\
& =\varphi(v) \cdot\left(2 \mathbf{d c}^{k-1} \cdot \mathbf{b}+\mathbf{c} \cdot \mathbf{b} \cdot(\mathbf{a}-\mathbf{b})^{k}+\mathbf{b} \cdot(\mathbf{a}-\mathbf{b})^{k+1}\right. \\
& \left.+\sum_{i+j=k-2} 2 \mathbf{d c ^ { i }} \cdot \mathbf{b} \cdot(\mathbf{a}-\mathbf{b})^{j+1}\right) .
\end{aligned}
$$

In order to simplify this expression, consider the butterfly poset of rank $k$. This is the poset consisting of two rank $i$ elements, for $i=1, \ldots, k-1$, adjoined with a minimal and maximal element. Each of the rank $i$ elements covers the rank $i-1$ element(s) for $i=1, \ldots, k-1$. The butterfly poset is the unique poset having the cd-index $\mathbf{c}^{k-1}$. It is also Eulerian. Applying (2.2) to the butterfly poset, we have

$$
\mathbf{c}^{k-1}=(\mathbf{a}-\mathbf{b})^{k-1}+2 \cdot \sum_{i+j=k-2} \mathbf{c}^{i} \cdot \mathbf{b} \cdot(\mathbf{a}-\mathbf{b})^{j} .
$$


Using this relation to simplify (3.3), we obtain

$$
\begin{aligned}
\varphi_{t}\left(v \cdot \mathbf{a b}^{k} \cdot x\right)-\kappa\left(v \cdot \mathbf{a b}^{k} \cdot x\right) & =\varphi(v) \cdot \mathbf{d} \cdot \mathbf{c}^{k} \\
& =1 / 2 \cdot \omega\left(v \cdot \mathbf{a b}^{k+1}\right) .
\end{aligned}
$$

By combining Lemmas 3.19 and 3.20, we have the following proposition.

Proposition 3.21 For an ab-monomial $v$ that begins with the letter $\mathbf{a}$, the following holds:

$$
\varphi_{t}(v)=\kappa(v)+1 / 2 \cdot \omega\left(H^{\prime}(v) \cdot \mathbf{b}\right) .
$$

We now obtain the main result for computing the ab-index of the face poset of a toric arrangement.

Theorem 3.22 Let $\mathcal{H}$ be a toric hyperplane arrangement on the $n$-dimensional torus $T^{n}$ that subdivides the torus into a regular cell complex. Then the ab-index of the face poset $T_{t}$ can be computed from the $\mathbf{a b}$-index of the intersection poset $\mathcal{P}$ as follows:

$$
\Psi\left(T_{t}\right)=(\mathbf{a}-\mathbf{b})^{n+1}+\frac{1}{2} \cdot \omega\left(\mathbf{a} \cdot H^{\prime}(\Psi(\mathcal{P})) \cdot \mathbf{b}\right)^{*} .
$$

Observe that in Lemmas 3.19 and 3.20, Proposition 3.21, and Theorem 3.22 no rational coefficients were introduced. Only the $\mathbf{a b}$-monomial $\mathbf{a}^{n}$ is mapped to a cdpolynomial with an odd coefficient, hence $1 / 2 \cdot \omega(v \cdot \mathbf{b})$ has all integer coefficients.

Continuation of Example 3.3 The flag $f$-vector of the intersection poset $\mathcal{P}$ in Example 3.3 is given by $\left(f_{\emptyset}, f_{1}, f_{2}, f_{12}\right)=(1,3,7,15)$, the flag $h$-vector by $\left(h_{\emptyset}, h_{1}, h_{2}, h_{12}\right)=(1,2,6,6)$, and so the ab-index is $\Psi(P)=\mathbf{a}^{2}+2 \cdot \mathbf{b a}+6 \cdot \mathbf{a b}+$ $6 \cdot \mathbf{b}^{2}$. Thus

$$
\begin{aligned}
\Psi\left(T_{t}\right) & =(\mathbf{a}-\mathbf{b})^{3}+1 / 2 \cdot \omega\left(\mathbf{a} \cdot H^{\prime}\left(\mathbf{a}^{2}+2 \cdot \mathbf{b a}+6 \cdot \mathbf{a b}+6 \cdot \mathbf{b}^{2}\right) \cdot \mathbf{b}\right)^{*} \\
& =(\mathbf{a}-\mathbf{b})^{3}+1 / 2 \cdot \omega(\mathbf{a} \cdot(7 \cdot \mathbf{a}+8 \cdot \mathbf{b}) \cdot \mathbf{b})^{*} \\
& =(\mathbf{a}-\mathbf{b})^{3}+1 / 2 \cdot \omega\left(7 \cdot \mathbf{a}^{2} \mathbf{b}+8 \cdot \mathbf{a} \mathbf{b}^{2}\right)^{*} \\
& =(\mathbf{a}-\mathbf{b})^{3}+7 \cdot \mathbf{d c}+8 \cdot \mathbf{c d},
\end{aligned}
$$

which agrees with the calculation in Example 3.3.

Theorem 3.22 gives a different approach from Corollary 3.12 for determining the $f$-vector of $T_{t}$. For notational ease, for positive integers $i$ and $j$, let $[i, j]=\{i, i+$ $1, \ldots, j\}$ and $[j]=\{1, \ldots, j\}$.

Corollary 3.23 The number of $i$-dimensional regions in the subdivision $T_{t}$ of the $n$-dimensional torus is given by the following sum of flag h-vector entries from the intersection poset $\mathcal{P}$ :

$$
f_{i+1}\left(T_{t}\right)=h_{[n-i, n]}(\mathcal{P})+h_{[n-i, n-1]}(\mathcal{P})+h_{[n-i+1, n]}(\mathcal{P})+h_{[n-i+1, n-1]}(\mathcal{P})
$$


for $1 \leq i \leq n-1$. The number of vertices is given by $f_{1}\left(T_{t}\right)=1+h_{n}(\mathcal{P})$ and the number of maximal regions by $f_{n+1}\left(T_{t}\right)=h_{[n-1]}(\mathcal{P})+h_{[n]}(\mathcal{P})$.

Proof Let $\langle\cdot \mid \cdot\rangle$ denote the inner product on $\mathbb{Z}\langle\mathbf{a}, \mathbf{b}\rangle$ defined by $\langle u \mid v\rangle=\delta_{u, v}$ for two ab-monomials $u$ and $v$. For $1 \leq i \leq n-1$, we have

$$
\begin{aligned}
f_{i+1}\left(T_{t}\right)= & 1+h_{i+1}\left(T_{t}\right) \\
= & 1+\left\langle\mathbf{a}^{i} \mathbf{b} \mathbf{a}^{n-i} \mid \Psi\left(T_{t}\right)\right\rangle \\
= & \frac{1}{2} \cdot\left\langle\mathbf{a}^{i} \mathbf{b} \mathbf{a}^{n-i} \mid \omega\left(\mathbf{a} \cdot H^{\prime}(\Psi(\mathcal{P})) \cdot \mathbf{b}\right)^{*}\right\rangle \\
= & \frac{1}{2} \cdot\left[\mathbf{c}^{i-1} \mathbf{d c}^{n-i}\right] \omega\left(\mathbf{a} \cdot H^{\prime}(\Psi(\mathcal{P})) \cdot \mathbf{b}\right)^{*} \\
& +\frac{1}{2} \cdot\left[\mathbf{c}^{i} \mathbf{d c ^ { n - i - 1 }}\right] \omega\left(\mathbf{a} \cdot H^{\prime}(\Psi(\mathcal{P})) \cdot \mathbf{b}\right)^{*} \\
= & \left\langle\mathbf{a}^{n-i} \cdot \mathbf{a b} \cdot \mathbf{b}^{i-1}+\mathbf{a}^{n-i-1} \cdot \mathbf{a b} \cdot \mathbf{b}^{i} \mid \mathbf{a} \cdot H^{\prime}(\Psi(\mathcal{P})) \cdot \mathbf{b}\right\rangle \\
= & \left\langle\mathbf{a}^{n-i-1} \cdot(\mathbf{a}+\mathbf{b}) \cdot \mathbf{b}^{i-1} \mid H^{\prime}(\Psi(\mathcal{P}))\right\rangle \\
= & \left\langle\mathbf{a}^{n-i-1} \cdot(\mathbf{a}+\mathbf{b}) \cdot \mathbf{b}^{i-1} \cdot(\mathbf{a}+\mathbf{b}) \mid \Psi(\mathcal{P})\right\rangle .
\end{aligned}
$$

Expanding in terms of the flag $h$-vector the result follows. The expressions for $f_{1}$ and $f_{n+1}$ are obtained by similar calculations.

The fact that Corollaries 3.12 and 3.23 are equivalent follows from the coalgebraic techniques in Theorem 2.5 .

\section{The Complex of Unbounded Regions}

\subsection{Zaslavsky and Bayer-Sturmfels}

The unbounded Zaslavsky invariant is defined by

$$
Z_{u b}(P)=Z(P)-2 \cdot Z_{b}(P) .
$$

As the name suggests, the number of unbounded regions in a noncentral arrangement is given by this invariant. By taking the difference of the two statements in Theorem 2.2 part (ii), we immediately have the following result.

Lemma 4.1 For a noncentral hyperplane arrangement $\mathcal{H}$, the number of unbounded regions is given by $Z_{u b}(\mathcal{L})$, where $\mathcal{L}$ is the intersection lattice of the arrangement $\mathcal{H}$.

Let $\mathcal{H}$ be a noncentral hyperplane arrangement in $\mathbb{R}^{n}$ with intersection lattice $\mathcal{L}$ having the empty set $\varnothing$ as the maximal element. Let $\mathcal{L}_{u b}$ denote the unbounded intersection lattice, that is, the subposet of the intersection lattice consisting of all affine subspaces with the points (dimension zero affine subspaces) omitted but with the 
empty set $\varnothing$ continuing to be the maximal element. Equivalently, the poset $\mathcal{L}_{u b}$ is the rank-selected poset $\mathcal{L}([1, n-1])$, that is, the poset $\mathcal{L}$ with the coatoms removed.

Let $T$ be the face lattice of the arrangement $\mathcal{H}$ with the minimal element $\hat{0}$ denoting the empty face and the maximal element denoted by $\hat{1}$. Similarly, let $T_{u b}$ denote the set of all faces in the face lattice $T$ which are not bounded. Observe that $T_{u b}$ includes the minimal and maximal elements of $T$ and that $T_{u b}$ is the face poset of an $(n-1)$-dimensional sphere. Pick $R$ large enough so that all of the bounded faces are strictly inside a ball of radius $R$. Intersect the arrangement $\mathcal{H}$ with a sphere of radius $R$. The resulting cell complex has face poset $T_{u b}$. Our goal is to compute the cd-index of $T_{u b}$ in terms of the ab-index of $\mathcal{L}_{u b}$.

The collection of unbounded faces of the arrangement $\mathcal{H}$ forms a lower order ideal in the poset $T^{*}$. Let $Q$ be the subposet of $T^{*}$ consisting of this ideal with a maximal element $\hat{1}$ adjoined. We define the rank of an element in $Q$ to be its rank in the original poset $T^{*}$, that is, for $x \in Q$, let $\rho_{Q}(x)=\rho_{T^{*}}(x)$. This rank convention will simplify the later arguments. As posets $T_{u b}^{*}$ and $Q$ are isomorphic. However, since their rank functions differ, their ab-indexes satisfy $\Psi\left(T_{u b}\right)^{*} \cdot(\mathbf{a}-\mathbf{b})=\Psi(Q)$.

Restrict the zero map $z: T^{*} \rightarrow \mathcal{L} \cup\{\hat{0}\}$ to form the map $z_{u b}: Q \rightarrow \mathcal{L} \cup\{\hat{0}\}$. Note that the map $z_{u b}$ is order- and rank-preserving, but not necessarily surjective. As before, we view the map $z_{u b}$ as a map from the set of chains of $Q$ to the set of chains of $\mathcal{L} \cup\{\hat{0}\}$. We have the following theorem, which is analogous to the BayerSturmfels result (Theorem 2.3).

Theorem 4.2 Let $\mathcal{H}$ be a noncentral hyperplane arrangement with intersection lattice $\mathcal{L}$. Let $c=\left\{\hat{0}=x_{0}<x_{1}<\cdots<x_{k}=\hat{1}\right\}$ be a chain in $\mathcal{L} \cup\{\hat{0}\}$ with $k \geq 2$. Then the cardinality of the inverse image of the chain $c$ under $z_{u b}$ is given by

$$
\left|z_{u b}^{-1}(c)\right|=\prod_{i=2}^{k-1} Z\left(\left[x_{i-1}, x_{i}\right]\right) \cdot Z_{u b}\left(\left[x_{k-1}, x_{k}\right]\right)
$$

Proof We need to count the number of ways we can select a chain $d=\left\{\hat{0}=y_{0}<\right.$ $\left.y_{1}<\cdots<y_{k}=\hat{1}\right\}$ in the poset of unbounded regions $Q$ such that $z_{u b}\left(y_{i}\right)=x_{i}$. The number of ways to select the element $y_{k-1}$ in $Q$ is the number of unbounded regions in the arrangement restricted to the subspace $x_{k-1}$. By Lemma 4.1 this can be done in $Z_{u b}\left(\left[x_{k-1}, x_{k}\right]\right)$ number of ways. Since $y_{k-1}$ is an unbounded face of the arrangement and all other elements in the chain $d$ contain the face $y_{k-1}$, the other elements must be unbounded.

The remainder of the proof is the same as that of Theorem 3.13.

Corollary 4.3 The flag $f$-vector entry $f_{S}\left(T_{u b}\right)$ is divisible by $2^{|S|}$ for any index set $S \subseteq\{1, \ldots, n\}$.

Proof The proof is the same as Corollary 3.14 with the extra observation that the Zaslavsky invariant $Z_{u b}$ is even. 
4.2 The Connection between Posets and Coalgebras

Define $\lambda_{u b}$ by $\lambda_{u b}=\eta-2 \cdot \beta$. By (2.5) and (2.6), for a graded poset $P$ we have

$$
\lambda_{u b}(\Psi(P))=Z_{u b}(P) \cdot(\mathbf{a}-\mathbf{b})^{\rho(P)-1} .
$$

Define a sequence of functions $\varphi_{u b, k}: \mathbb{Z}\langle\mathbf{a}, \mathbf{b}\rangle \rightarrow \mathbb{Z}\langle\mathbf{a}, \mathbf{b}\rangle$ by $\varphi_{u b, 1}=\kappa$ and for $k>1$,

$$
\varphi_{u b, k}(v)=\sum_{v} \kappa\left(v_{(1)}\right) \cdot \mathbf{b} \cdot \eta\left(v_{(2)}\right) \cdot \mathbf{b} \cdot \eta\left(v_{(3)}\right) \cdot \mathbf{b} \cdots \mathbf{b} \cdot \eta\left(v_{(k-1)}\right) \cdot \mathbf{b} \cdot \lambda_{u b}\left(v_{(k)}\right)
$$

Finally, let $\varphi_{u b}(v)$ be the sum $\varphi_{u b}(v)=\sum_{k \geq 1} \varphi_{u b, k}(v)$.

Similar to Theorem 3.17 we have the next result. The proof only differs in replacing the map $z_{t}: T_{t}^{*} \rightarrow \mathcal{P} \cup\{\hat{0}\}$ with $z_{u b}: Q \rightarrow \mathcal{L} \cup\{\hat{0}\}$ and the invariant $Z_{t}$ by $Z_{u b}$.

Theorem 4.4 The ab-index of the poset $Q$ of unbounded regions of a noncentral arrangement is given by

$$
\Psi(Q)=\varphi_{u b}(\Psi(\mathcal{L} \cup\{\hat{0}\}))
$$

\subsection{Evaluating the Function $\varphi_{u b}$}

In this subsection we analyze the behavior of $\varphi_{u b}$.

Lemma 4.5 For any ab-monomial $v$,

$$
\varphi_{u b}(v)=\varphi(v)-2 \cdot \sum_{v} \varphi\left(v_{(1)}\right) \cdot \mathbf{b} \cdot \beta\left(v_{(2)}\right) .
$$

Proof Using the coassociative identity $\Delta^{k-1}=\left(\Delta^{k-2} \otimes\right.$ id $) \circ \Delta$, we have for $k \geq 2$,

$$
\begin{aligned}
\varphi_{u b, k}(v) & =\varphi_{k}(v)-2 \cdot \sum_{v} \kappa\left(v_{(1)}\right) \cdot \mathbf{b} \cdot \eta\left(v_{(2)}\right) \cdot \mathbf{b} \cdots \mathbf{b} \cdot \eta\left(v_{(k-1)}\right) \cdot \mathbf{b} \cdot \beta\left(v_{(k)}\right) \\
& =\varphi_{k}(v)-2 \cdot \sum_{v} \sum_{v_{(1)}} \kappa\left(v_{(1,1)}\right) \cdot \mathbf{b} \cdot \eta\left(v_{(1,2)}\right) \cdot \mathbf{b} \cdots \mathbf{b} \cdot \eta\left(v_{(1, k-1)}\right) \cdot \mathbf{b} \cdot \beta\left(v_{(2)}\right) \\
& =\varphi_{k}(v)-2 \cdot \sum_{v} \varphi_{k-1}\left(v_{(1)}\right) \cdot \mathbf{b} \cdot \beta\left(v_{(2)}\right) .
\end{aligned}
$$

The result then follows by summing over all $k \geq 2$ and adding $\varphi_{u b, 1}(v)=\kappa(v)=$ $\varphi_{1}(v)$.

Lemma 4.6 Let $v$ be an ab-monomial. Then

$$
\varphi_{u b}(v \cdot \mathbf{a})=\varphi(v) \cdot(\mathbf{a}-\mathbf{b}) .
$$


Proof By Lemma 4.5 and the Newtonian relation (2.3) we have

$$
\varphi_{u b}(v \cdot \mathbf{a})=\varphi(v \cdot \mathbf{a})-2 \cdot \varphi(v) \cdot \mathbf{b} \cdot \beta(1)-2 \cdot \sum_{v} \varphi\left(v_{(1)}\right) \cdot \mathbf{b} \cdot \beta\left(v_{(2)} \cdot \mathbf{a}\right) .
$$

By (2.7), $\varphi(v \cdot \mathbf{a})=\varphi(v) \cdot \mathbf{c}$. The summation above is zero because $\beta\left(v_{(2)} \cdot \mathbf{a}\right)$ is always zero. Hence $\varphi_{u b}(v \cdot \mathbf{a})=\varphi(v) \cdot(\mathbf{c}-2 \mathbf{b})=\varphi(v) \cdot(\mathbf{a}-\mathbf{b})$.

Lemma 4.7 Let $v$ be an ab-monomial. Then

$$
\varphi_{u b}(v \cdot \mathbf{b b})=\varphi_{u b}(v \cdot \mathbf{b}) \cdot(\mathbf{a}-\mathbf{b}) .
$$

Proof Let $u=v \cdot \mathbf{b}$. Applying Lemma 4.5 and the Newtonian relation (2.3) to $u$ gives

$$
\begin{aligned}
\varphi_{u b}(u \cdot \mathbf{b}) & =\varphi(u \cdot \mathbf{b})-2 \cdot \varphi(u) \cdot \mathbf{b} \cdot \beta(1)-2 \cdot \sum_{u} \varphi\left(u_{(1)}\right) \cdot \mathbf{b} \cdot \beta\left(u_{(2)} \cdot \mathbf{b}\right) \\
& =\varphi(u) \cdot(\mathbf{c}-2 \mathbf{b})-2 \cdot \sum_{u} \varphi\left(u_{(1)}\right) \cdot \mathbf{b} \cdot \beta\left(u_{(2)}\right) \cdot(\mathbf{a}-\mathbf{b}) \\
& =\left(\varphi(u)-2 \cdot \sum_{u} \varphi\left(u_{(1)}\right) \cdot \mathbf{b} \cdot \beta\left(u_{(2)}\right)\right) \cdot(\mathbf{a}-\mathbf{b}) \\
& =\varphi_{u b}(u) \cdot(\mathbf{a}-\mathbf{b}) .
\end{aligned}
$$

Here we have used the two facts $\varphi(u \cdot \mathbf{b})=\varphi(u) \cdot \mathbf{c}$ and $\beta\left(u_{(2)} \cdot \mathbf{b}\right)=\beta\left(u_{(2)}\right)$. $(\mathbf{a}-\mathbf{b})$.

Lemma 4.8 Let $v$ be an ab-monomial. Then $\varphi_{u b}(v \cdot \mathbf{a b})=0$.

Proof Directly we have

$$
\begin{aligned}
\varphi_{u b}(v \cdot \mathbf{a b})= & \varphi(v \cdot \mathbf{a b})-2 \cdot \varphi(v) \cdot \mathbf{b} \cdot \beta(\mathbf{b})-2 \cdot \varphi(v \cdot \mathbf{a}) \cdot \mathbf{b} \cdot \beta(1) \\
& -2 \cdot \sum_{v} \varphi\left(v_{(1)}\right) \cdot \mathbf{b} \cdot \beta\left(v_{(2)} \cdot \mathbf{a b}\right) \\
= & \varphi(v) \cdot 2 \mathbf{d}-2 \cdot \varphi(v) \cdot \mathbf{b} \cdot(\mathbf{a}-\mathbf{b})-2 \cdot \varphi(v) \cdot \mathbf{c b} \\
= & 2 \cdot \varphi(v) \cdot(\mathbf{d}-\mathbf{b}(\mathbf{a}-\mathbf{b})-\mathbf{c b}) \\
= & 0
\end{aligned}
$$

where we have used the facts $\varphi(v \cdot \mathbf{a b})=\varphi(v) \cdot 2 \mathbf{d}$ and $\beta\left(v_{(2)} \cdot \mathbf{a b}\right)=0$.

The previous three lemmas enable us to determine $\varphi_{u b}$. In order to obtain more compact notation, define the map $r: \mathbb{Z}\langle\mathbf{a}, \mathbf{b}\rangle \rightarrow \mathbb{Z}\langle\mathbf{a}, \mathbf{b}\rangle$ by $r(1)=0, r(v \cdot \mathbf{a})=v$, and $r(v \cdot \mathbf{b})=0$. By using the chain definition of the ab-index, it is straightforward to see that $\Psi\left(\mathcal{L}_{u b}\right)=r(\Psi(\mathcal{L}))$. 
Proposition 4.9 Let $w$ be an ab-polynomial homogeneous of degree greater than zero. Then

$$
\varphi_{u b}(\mathbf{a} \cdot w)=\omega(\mathbf{a} \cdot r(w)) \cdot(\mathbf{a}-\mathbf{b}) .
$$

Proof The case $w=v \cdot \mathbf{a}$ follows from Lemma 4.6. The remaining case is $w=$ $v \cdot \mathbf{b}$. Note that $\mathbf{a} \cdot v \cdot \mathbf{b}$ can be factored as $u \cdot \mathbf{a b} \cdot \mathbf{b}^{k}$ for a monomial $u$. Hence $\varphi_{u b}\left(u \cdot \mathbf{a b} \cdot \mathbf{b}^{k}\right)=\varphi_{u b}(u \cdot \mathbf{a b}) \cdot(\mathbf{a}-\mathbf{b})^{k}=0$ by Lemmas 4.7 and 4.8 .

We combine all these results to conclude that the cd-index of the poset of unbounded regions $T_{u b}$ can be computed in terms of the ab-index of the unbounded intersection lattice $\mathcal{L}_{u b}$.

Theorem 4.10 Let $\mathcal{H}$ be a noncentral hyperplane arrangement with the unbounded intersection lattice $\mathcal{L}_{u b}$ and poset of unbounded regions $T_{u b}$. Then the ab-index of $T_{u b}$ is given by

$$
\Psi\left(T_{u b}\right)=\omega\left(\mathbf{a} \cdot \Psi\left(\mathcal{L}_{u b}\right)\right)^{*}
$$

Proof We have that

$$
\begin{aligned}
\Psi\left(T_{u b}\right)^{*} \cdot(\mathbf{a}-\mathbf{b}) & =\Psi(Q) \\
& =\varphi_{u b}(\mathbf{a} \cdot \Psi(\mathcal{L})) \\
& =\omega(\mathbf{a} \cdot r(\Psi(\mathcal{L}))) \cdot(\mathbf{a}-\mathbf{b}) \\
& =\omega\left(\mathbf{a} \cdot \Psi\left(\mathcal{L}_{u b}\right)\right) \cdot(\mathbf{a}-\mathbf{b}) .
\end{aligned}
$$

By cancelling $\mathbf{a}-\mathbf{b}$ on both sides of this identity, the result follows.

Example 4.11 Consider the noncentral hyperplane arrangement consisting of the six hyperplanes $x=0,1, y=0,1$, and $z=0$, 1 . See Fig. 3. After intersecting this arrangement with a sphere of large enough radius, we obtain the cell complex in Fig. 4. The polytopal realization of this complex is known as the rhombicuboctahedron. The dual of the face lattice of this spherical complex is not realized by a zonotope. However, one can view the dual lattice as the face lattice of a $2 \times 2 \times 2$ pile of cubes.

The intersection lattice $\mathcal{L}$ is the face lattice of the three-dimensional crosspolytope, in other words, the octahedron. Hence the lattice of unbounded intersections $\mathcal{L}_{u b}$ has the flag $f$-vector $\left(f_{\varnothing}, f_{1}, f_{2}, f_{12}\right)=(1,6,12,24)$ and the flag $h$-vector $\left(h_{\emptyset}, h_{1}, h_{2}, h_{12}\right)=(1,5,11,7)$. The $\mathbf{a b}$-index is given by $\Psi\left(\mathcal{L}_{u b}\right)=\mathbf{a}^{2}+5 \cdot \mathbf{b a}+$ $11 \cdot \mathbf{a b}+7 \cdot \mathbf{b}^{2}$. Hence the $\mathbf{c d}$-index of $T_{u b}$ is

$$
\begin{aligned}
\Psi\left(T_{u b}\right) & =\omega\left(\mathbf{a}^{3}+5 \cdot \mathbf{a b a}+11 \cdot \mathbf{a}^{2} \mathbf{b}+7 \cdot \mathbf{a b}^{2}\right)^{*} \\
& =\mathbf{c}^{3}+22 \cdot \mathbf{d c}+24 \cdot \mathbf{c d} .
\end{aligned}
$$


Fig. 3 The noncentral arrangement $x, y, z=0,1$

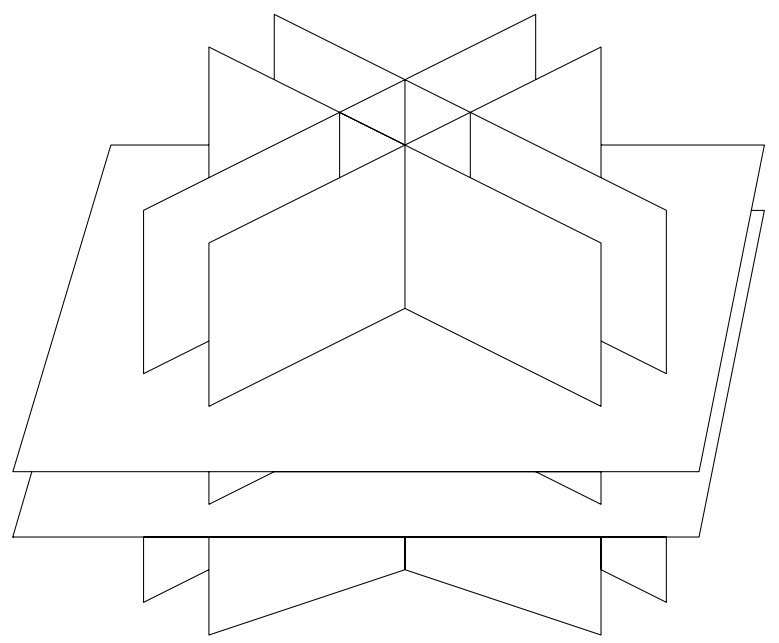

Fig. 4 The spherical subdivision obtained from the noncentral arrangement $x, y, z=0,1$

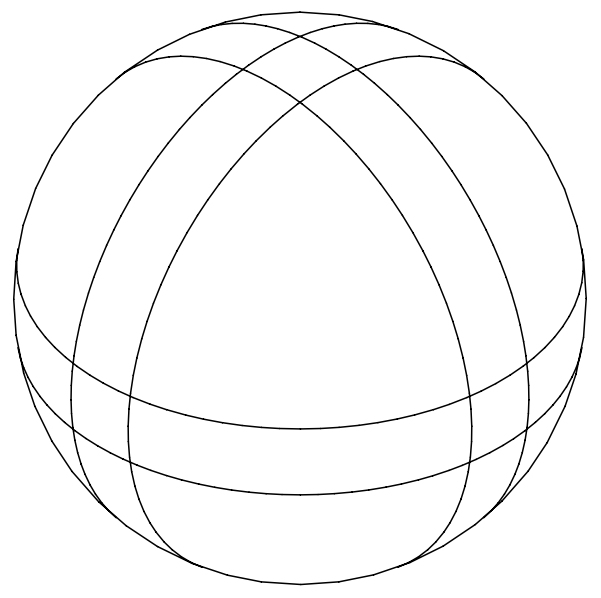

\section{Concluding Remarks}

For regular subdivisions of manifolds, there is now a plethora of questions to ask.

(i) What is the right analogue of a regular subdivision in order that it be polytopal? Can flag $f$-vectors be classified for polytopal subdivisions?

(ii) Is there a Kalai convolution for manifolds that will generate more inequalities for flag $f$-vectors? [33]

(iii) Is there a lifting technique that will yield more inequalities for higher dimensional manifolds? [17]

(iv) Are there minimization inequalities for the cd-coefficients in the polynomial $\Psi$ ? As a first step, can one prove the nonnegativity of $\Psi$ ? $[7,18]$

(v) Is there an extension of the toric $g$-inequalities to manifolds? [4, 32, 34, 42] 
(vi) Can the coefficients for $\Psi$ be minimized for regular toric arrangements as was done in the case of central hyperplane arrangements? [8]

The most straightforward manifold to study is the $n$-dimensional projective space $P^{n}$. We offer the following result in obtaining the ab-index of subdivisions of $P^{n}$.

Theorem 5.1 Let $\Omega$ be a centrally symmetric regular subdivision of the $n$-dimensional sphere $S^{n}$. Assume that when antipodal points of the sphere are identified, a regular subdivision $\Omega^{\prime}$ of the projective space $P^{n}$ is obtained. Then the $\mathbf{a b}$-index of $\Omega^{\prime}$ is given by

$$
\Psi\left(\Omega^{\prime}\right)=\frac{\mathbf{c}^{n+1}+(\mathbf{a}-\mathbf{b})^{n+1}}{2}+\frac{\Phi}{2},
$$

where the cd-index of $\Omega$ is $\Psi(\Omega)=\mathbf{c}^{n+1}+\Phi$.

Proof Each chain $c=\left\{\hat{0}=x_{0}<x_{1}<\cdots<x_{k}=\hat{1}\right\}$ with $k \geq 2$ in $\Omega^{\prime}$ corresponds to two chains in $\Omega$ with the same weight $\operatorname{wt}(c)$. The chain $c=\left\{\hat{0}=x_{0}<x_{1}=\hat{1}\right\}$ corresponds to exactly one chain in $\Omega$ and has weight $(\mathbf{a}-\mathbf{b})^{n+1}$. Hence $\Psi(\Omega)=$ $2 \cdot \Psi\left(\Omega^{\prime}\right)-(\mathbf{a}-\mathbf{b})^{n+1}$, proving the result.

The results in this paper have been stated for hyperplane arrangements. In true generality one could work with the underlying oriented matroid, especially since there are nonrealizable ones such as the non-Pappus oriented matroid. All of these can be represented as pseudo-hyperplane arrangements. However, we have chosen to work with hyperplane arrangements in order not to lose the geometric intuition.

Poset transformations related to the $\omega$ map appear in [16, 23, 29]. Are there toric or affine analogues of these poset transforms?

Another way to encode the flag $f$-vector data of a poset is to use the quasisymmetric function of a poset [14]. In this language the $\omega$ map is translated to Stembridge's $\vartheta$ map; see $[9,44]$. Would the results of Theorems 3.22 and 4.10 be appealing in the quasisymmetric function viewpoint?

Richard Stanley has asked if the coefficients of the toric characteristic polynomial are alternating. If so, is there any combinatorial interpretation of the absolute values of the coefficients?

A far reaching generalization of Zaslavsky's results for hyperplane arrangements is by Goresky and MacPherson [25]. Their results determine the cohomology groups of the complement of a complex hyperplane arrangement. For a toric analogue of the Goresky-MacPherson results, see the work of De Concini and Procesi [12]. For algebraic considerations of toric arrangements, see [13, 36-38].

Greene and Zaslavsky [26] also give an interpretation of the derivative of the chromatic polynomial of a graph evaluated at 1 . Given an edge $i j$ of a graph $G$, the number of acyclic orientations of the graph with unique source at $i$ and unique sink at $j$ is given by $\left.(-1)^{n} \cdot \frac{d}{d t} \chi(G ; t)\right|_{t=1}$. Is there a geometric proof of this fact analogous to the proof of Theorem 3.10?

In Sect. 3 we restricted ourselves to studying arrangements that cut the torus into regular cell complexes. In a future paper [24], two of the authors are developing the notion of a cd-index for nonregular cell complexes. 
Acknowledgements The authors thank the MIT Mathematics Department where this research was carried out. We also thank the referee for his careful comments and for bringing the 1977 paper of Zaslavsky to our attention, Andrew Klapper for his suggestions to improve the exposition, and Tricia Hersh for suggesting that we look at graphical arrangements on the torus. The first and third authors were partially supported by National Security Agency grant H98230-06-1-0072.

\section{References}

1. Athanasiadis, C.A.: Characteristic polynomials of subspace arrangements and finite fields. Adv. Math. 122, 193-233 (1996)

2. Athanasiadis, C.A.: Extended Linial hyperplane arrangements for root systems and a conjecture of Postnikov and Stanley. J. Algebraic Combin. 10, 207-225 (1999)

3. Bayer, M., Billera, L.: Generalized Dehn-Sommerville relations for polytopes, spheres and Eulerian partially ordered sets. Invent. Math. 79, 143-157 (1985)

4. Bayer, M., Ehrenborg, R.: The toric $h$-vectors of partially ordered sets. Trans. Amer. Math. Soc. 352, 4515-4531 (2000)

5. Bayer, M., Klapper, A.: A new index for polytopes. Discrete Comput. Geom. 6, 33-47 (1991)

6. Bayer, M., Sturmfels, B.: Lawrence polytopes. Can. J. Math. 42, 62-79 (1990)

7. Billera, L.J., Ehrenborg, R.: Monotonicity of the cd-index for polytopes. Math. Z. 233, 421-441 (2000)

8. Billera, L.J., Ehrenborg, R., Readdy, M.: The c-2d-index of oriented matroids. J. Combin. Theory Ser. A 80, 79-105 (1997)

9. Billera, L.J., Hsiao, S.K., van Willigenburg, S.: Peak quasisymmetric functions and Eulerian enumeration. Adv. Math. 176, 248-276 (2003)

10. Björner, A.: Topological Methods. Handbook of Combinatorics, vol. 2, pp. 1819-1872. Elsevier, Amsterdam (1995)

11. Chen, B.: On characteristic polynomials of subspace arrangements. J. Combin. Theory Ser. A 90, 347-352 (2000)

12. De Concini, C., Procesi, C.: On the geometry of toric arrangements. Transform. Groups 10, 387-422 (2005)

13. Douglass, J.M.: Toral arrangements and hyperplane arrangements. Rocky Mountain J. Math. 28, 939956 (1998)

14. Ehrenborg, R.: On posets and Hopf algebras. Adv. Math. 119, 1-25 (1996)

15. Ehrenborg, R.: $k$-Eulerian posets. Order 18, 227-236 (2001)

16. Ehrenborg, R.: The $r$-signed Birkhoff transform. Preprint (2004)

17. Ehrenborg, R.: Lifting inequalities for polytopes. Adv. Math. 193, 205-222 (2005)

18. Ehrenborg, R., Karu, K.: Decomposition theorem for the cd-index of Gorenstein* posets. J. Algebraic Combin. 26, 225-251 (2007)

19. Ehrenborg, R., Readdy, M.: Coproducts and the cd-index. J. Algebraic Combin. 8, 273-299 (1998)

20. Ehrenborg, R., Readdy, M.: On valuations, the characteristic polynomial and complex subspace arrangements. Adv. Math. 134, 32-42 (1998)

21. Ehrenborg, R., Readdy, M.: The Dowling transform of subspace arrangements. J. Combin. Theory Ser. A 91, 322-333 (2000)

22. Ehrenborg, R., Readdy, M.: Homology of Newtonian coalgebras. European J. Combin. 23, 919-927 (2002)

23. Ehrenborg, R., Readdy, M.: The Tchebyshev transforms of the first and second kind. Ann. Comb. (to appear)

24. Ehrenborg, R., Slone, M.: The cd-index of non-regular cell complexes (in preparation)

25. Goresky, M., MacPherson, R.: Stratified Morse Theory. Ergebnisse der Mathematik und ihrer Grenzgebiete (3) (Results in Mathematics and Related Areas (3)), vol. 14. Springer, Berlin (1988)

26. Greene, C., Zaslavsky, T.: On the interpretation of Whitney numbers through arrangements of hyperplanes, zonotopes, non-Radon partitions, and orientations of graphs. Trans. Amer. Math. Soc. 280, 97-126 (1983)

27. Groemer, H.: On the extension of additive functionals on classes of convex sets. Pacific J. Math. 75, 397-410 (1978)

28. Grünbaum, B.: Convex Polytopes, 2nd edn. Springer, New York (2003) 
29. Hsiao, S.K.: A signed analog of the Birkhoff transform. J. Combin. Theory Ser. A 113, 251-272 (2006)

30. Joni, S.A., Rota, G.-C.: Coalgebras and bialgebras in combinatorics. Stud. Appl. Math. 61, 93-139 (1979)

31. Józefiak, T., Sagan, B.: Basic derivations for subarrangements of Coxeter arrangements. J. Algebraic Combin. 2, 291-320 (1993)

32. Kalai, G.: Rigidity and the lower bound theorem. I. Invent. Math. 88, 125-151 (1987)

33. Kalai, G.: A new basis of polytopes. J. Combin. Theory Ser. A 49, 191-209 (1988)

34. Karu, K.: Hard Lefschetz theorem for nonrational polytopes. Invent. Math. 157, 419-447 (2004)

35. Klain, D., Rota, G.-C.: Introduction to Geometric Probability. Lezioni Lincee. Cambridge University Press, Cambridge (1997)

36. Macmeikan, C.: The Poincaré polynomial of an MP arrangement. Proc. Amer. Math. Soc. 132, 15751580 (2004)

37. Macmeikan, C.: Modules of derivations for toral arrangements. Indag. Math. (N.S.) 15, 257-267 (2004)

38. Macmeikan, C.: Toral arrangements. In: The COE Seminar on Mathematical Sciences 2004. Sem. Math. Sci., vol. 31, pp. 37-54. Keio Univ., Yokohama (2004)

39. Novik, I., Postnikov, A., Sturmfels, B.: Syzygies of oriented matroids. Duke Math. J. 111, 287-317 (2002)

40. Stanley, R.P.: Acyclic orientations of graphs. Discrete Math. 5, 171-178 (1973)

41. Stanley, R.P.: Enumerative Combinatorics, vol. I. Wadsworth, Belmont (1986)

42. Stanley, R.P.: Generalized $h$-vectors, intersection cohomology of toric varieties, and related results. In: Commutative Algebra and Combinatorics, Kyoto, 1985. Adv. Stud. Pure Math., vol. 11, pp. 187213. North-Holland, Amsterdam (1987)

43. Stanley, R.P.: Flag $f$-vectors and the $c d$-index. Math. Z. 216, 483-499 (1994)

44. Stembridge, J.: Enriched $P$-partitions. Trans. Amer. Math. Soc. 349, 763-788 (1997)

45. Swartz, E.: Face enumeration: from spheres to manifolds. J. Eur. Math. Soc. (to appear)

46. Sweedler, M.: Hopf Algebras. Benjamin, New York (1969)

47. Zaslavsky, T.: Facing up to arrangements: face count formulas for partitions of space by hyperplanes. Mem. Amer. Math. Soc. 154 (1975)

48. Zaslavsky, T.: A combinatorial analysis of topological dissections. Adv. Math. 25, 267-285 (1977) 